\title{
Optimal Bandwidth and Power Allocation for Sum Ergodic Capacity under Fading Channels in Cognitive Radio Networks
}

\author{
Xiaowen Gong, Student Member, IEEE, Sergiy A. Vorobyov, Senior \\ Member, IEEE, and Chintha Tellambura, Senior Member, IEEE
}

\begin{abstract}
This paper studies optimal bandwidth and power allocation in a cognitive radio network where multiple secondary users (SUs) share the licensed spectrum of a primary user (PU) under fading channels using the frequency division multiple access scheme. The sum ergodic capacity of all the SUs is taken as the performance metric of the network. Besides all combinations of the peak/average transmit power constraints at the SUs and the peak/average interference power constraint imposed by the PU, total bandwidth constraint of the licensed spectrum is also taken into account. Optimal bandwidth allocation is derived in closed-form for any given power allocation. The structures of optimal power allocations are also derived under all possible combinations of the aforementioned power constraints. These structures indicate the possible numbers of users that transmit at nonzero power but below their corresponding peak powers, and show that other users do not transmit or transmit at their corresponding peak power. Based on these structures, efficient algorithms are developed for finding the optimal power allocations.
\end{abstract}

\section{Index Terms}

Bandwidth and power allocation, cognitive radio, fading channels, frequency division multiple access, sum ergodic capacity

This work was supported in parts by research grants from the Natural Science and Engineering Research Council (NSERC) of Canada and Alberta Ingenuity New Faculty Award.

The authors are with the Department of Electrical and Computer Engineering, University of Alberta, 9107-116 St., Edmonton, Alberta, T6G 2V4 Canada. The contacting emails are \{xgong2, vorobyov, chintha\}@ece.ualberta.ca.

Corresponding author: Sergiy A. Vorobyov, Dept. of Electrical and Computer Engineering, University of Alberta, 9107116 St., Edmonton, Alberta, T6G 2V4, Canada; Phone: +1 (780) 492 9702, Fax: +1 (780) 4921811. 


\section{INTRODUCTION}

Cognitive radio is a promising technology for improving spectrum utilization in wireless communications systems [1]. A secondary user (SU) in a cognitive radio network is allowed to access the licensed spectrum allocated to a primary user (PU) if the spectrum is not utilized by the PU. Such a spectrum sharing strategy, which is referred to as spectrum overlay or opportunistic spectrum access (OSA) [2], requires correct detection of spectrum opportunities by the SU. Existing works on spectrum overlay have mainly studied spectrum sensing and access policies at the medium access control (MAC) layer [3]- [6]. An alternative strategy, which is known as spectrum underlay [7]- [12], enables the PU and the SU to transmit simultaneously, provided that the received interference power by the PU is below a prescribed threshold level. A number of works have recently studied information theoretic limits for resource allocation in the context of spectrum underlay.

In [13], the optimal power allocation which aims at maximizing the ergodic capacity achieved by an SU is derived for various channel fading models subject to the peak interference power (PIP) constraint or average interference power (AIP) constraint imposed by a PU. In [14], the authors derive the optimal power allocation for the ergodic capacity, outage capacity, and minimum-rate capacity of an SU under both the PIP and AIP constraints from a PU. The ergodic capacity, delay-limited capacity, and outage capacity of an SU is studied in [15] under different combinations of the peak transmit power (PTP) constraint or average transmit power (ATP) constraint at the SU and the PIP constraint or AIP constraint from a PU. However, all the papers mentioned above consider the setup of a single SU. The most recent work [16] studies a cognitive radio network of multiple SUs under multiple access channel and broadcast channel models, where the optimal power allocation is derived to achieve the maximum sum ergodic capacity of the SUs subject to various mixed transmit and interference power constraints. The optimality conditions for the dynamic time division multiple access scheme are also derived.

In this paper, we focus on a cognitive radio network where multiple SUs share the licensed spectrum of a PU using the frequency division multiple access (FDMA) scheme. The sum ergodic capacity of the SUs, which is a relevant network performance metric for delay-tolerant traffics, is studied. Besides the transmit power constraints at the SUs and the interference power constraint imposed by the PU, which are also considered in [13]- [16], we also take into account the total 
bandwidth constraint of the shared spectrum. Such study is motivated by the fact observed for a number of different applications that joint bandwidth and power allocation can significantly improve the performance of systems with limited both individual (power) and public (bandwidth) resources [17]- [23]. Thus, in this paper, instead of conventional fixed and equal bandwidth allocation used in FDMA, we investigate dynamic and unequal bandwidth allocation, where the bandwidth allocation varies for different SUs at different channel fading states. Moreover, different from the existing works [13]- [16], all combinations of the transmit power constraints and the interference power constraints are considered, including both PTP and ATP constraints combined with both PIP and AIP constraints.

We first derive the optimal bandwidth allocation for any given power allocation in any channel fading state, which results in equivalent problems that only involve power allocation. Using the convexity of the resultant power allocation problems, we apply dual decomposition which transforms these problems into equivalent dual problems, where each dual function involves a power allocation subproblem associated with a specific channel fading state. The dual problems can be solved using standard subgradient algorithms. For the power allocation subproblem under all combinations of the power constraints, we derive the structures of the optimal power allocations. These structures indicate the possible numbers of users that transmit at nonzero power but below their corresponding peak powers, and show that other users do not transmit or transmit at their corresponding peak power. Based on these structures, we develop algorithms for finding the optimal power allocations in each channel fading state.

The rest of the paper is organized as follows. Section [I summarizes the system model and formulates corresponding sum ergodic capacity maximization problems. Section III derives the optimal bandwidth allocation for the problems formulated in Section \ subject to the bandwidth constraint. Section IV obtains the optimal power allocations from the resultant problems in Section III under all combinations of the transmit power constraints and interference power constraints. Numerical results for the maximum sum ergodic capacity under different combinations of the power constraints and the bandwidth constraint are shown in Section V. Section VI concludes the paper. 


\section{SySTEM MODEL}

Consider a cognitive radio network of $N$ SUs and one PU. The PU occupies a spectrum of bandwidth $W$ for its transmission, while the same spectrum is shared by the SUs. The spectrum is assumed to be divided into distinct and nonoverlapping flat fading channels with different bandwidth, so that the SUs share the spectrum through FDMA to avoid interferences with each other. The channel power gains between the $i$ th SU transmitter (SU-Tx) and the $i$ th SU receiver (SU-Rx) and between the $i$ th SU-Tx and the PU receiver (PU-Rx) are denoted by $h_{i}$ and $g_{i}$, respectively. The channel power gains, i.e., $\boldsymbol{g} \triangleq\left[\begin{array}{llll}g_{1} & g_{2} & \cdots & g_{N}\end{array}\right]$ and $\boldsymbol{h} \triangleq\left[\begin{array}{llll}h_{1} & h_{2} & \cdots & h_{N}\end{array}\right]$, are assumed to be drawn from an ergodic and stationary vector random process. We further assume that full channel state information (CSI), i.e., the joint probability density function (PDF) of the channel power gains and the instantaneous channel power gains, are known at the SUs. 1 The noise at each SU-Rx plus the interference from the PU transmitter (PU-Tx) is assumed to be additive white Gaussian noise (AWGN) with unit power spectral density (PSD).

We denote the transmit power of the $i$ th SU-Tx and the channel bandwidth allocated to the $i$ th SU-Tx as $p_{i}(\boldsymbol{g}, \boldsymbol{h})$ and $w_{i}(\boldsymbol{h}, \boldsymbol{g})$, respectively, for the instantaneous channel power gains $\boldsymbol{g}$ and $\boldsymbol{h}$. Then the total bandwidth constraint can be expressed as

$$
\sum_{i=1}^{N} w_{i}(\boldsymbol{h}, \boldsymbol{g}) \leq W, \forall \boldsymbol{h}, \boldsymbol{g} .
$$

The PTP constraints are given by

$$
p_{i}(\boldsymbol{h}, \boldsymbol{g}) \leq P_{i}^{p k}, \forall i, \boldsymbol{h}, \boldsymbol{g}
$$

where $P_{i}^{p k}$ denotes the maximum peak transmit power of the $i$ th SU-Tx. The PIP constraint is given by

$$
\sum_{i=1}^{N} g_{i} p_{i}(\boldsymbol{h}, \boldsymbol{g}) \leq Q^{p k}, \forall \boldsymbol{h}, \boldsymbol{g}
$$

where $Q^{p k}$ denotes the maximum peak interference power allowed at the PU-Rx. The ATP constraints are given by

$$
\mathrm{E}\left\{p_{i}(\boldsymbol{h}, \boldsymbol{g})\right\} \leq P_{i}^{a v}, \forall i
$$

${ }^{1}$ Note that the full CSI assumption is typical in the context of cognitive radio and is also made in other works such as [13][16]. Indeed, under this assumption we aim at investigating the information-theoretic limits on the sum ergodic capacity. 
where the expectation is taken over $\boldsymbol{h}$ and $\boldsymbol{g}$, and $P_{i}^{a v}$ denotes the maximum average transmit power of the $i$ th SU-Tx. The AIP constraint is given by

$$
\mathrm{E}\left\{\sum_{i=1}^{N} g_{i} p_{i}(\boldsymbol{h}, \boldsymbol{g})\right\} \leq Q^{a v}
$$

where $Q^{a v}$ denotes the maximum average interference power allowed at the PU-Rx.

The objective is to maximize the sum ergodic capacity of the SUs, which can be written as

$$
\max _{\left\{w_{i}(\boldsymbol{h}, \boldsymbol{g}), p_{i}(\boldsymbol{h}, \boldsymbol{g})\right\} \in \mathcal{F}} \mathrm{E}\left\{\sum_{i=1}^{N} w_{i}(\boldsymbol{h}, \boldsymbol{g}) \log \left(1+\frac{h_{i} p_{i}(\boldsymbol{h}, \boldsymbol{g})}{w_{i}(\boldsymbol{h}, \boldsymbol{g})}\right)\right\}
$$

where $\mathcal{F}$ is a feasible set specified by the bandwidth constraints (1) and a particular combination of the transmit power constraints $\{(2),(4)\}$ and the interference power constraints $\{(\sqrt[3]{3}),(5)\}$. Note that the constraints on nonnegativity of the bandwidth and power allocations, i.e., $w_{i}(\boldsymbol{h}, \boldsymbol{g}) \geq 0$ and $p_{i}(\boldsymbol{h}, \boldsymbol{g}) \geq 0, \forall i, \boldsymbol{h}, \boldsymbol{g}$, are natural and, thus, omitted through out the paper for brevity.

It can be shown that the objective function of the problem (6) is concave with respect to $\left\{w_{i}(\boldsymbol{h}, \boldsymbol{g}), p_{i}(\boldsymbol{h}, \boldsymbol{g})\right\}, \forall i, \boldsymbol{h}, \boldsymbol{g}$. It can also be seen that the bandwidth and power constraints (1)(5) are linear and, thus, convex. Therefore, the sum ergodic capacity maximization problem (6) under different combinations of the constraints (1)-(5) is a convex optimization problem.

\section{Optimal BANDWidth Allocation}

Given that the power allocation $p_{i}(\boldsymbol{h}, \boldsymbol{g}), \forall i, \boldsymbol{h}, \boldsymbol{g}$, is fixed, the maximum sum ergodic capacity can be expressed as $\mathrm{E}\left\{f_{0}(\boldsymbol{h}, \boldsymbol{g})\right\}$, where $f_{0}(\boldsymbol{h}, \boldsymbol{g})$ is given by

$$
\begin{aligned}
f_{0}(\boldsymbol{h}, \boldsymbol{g}) \triangleq \quad & \max _{\left\{w_{i}(\boldsymbol{h}, \boldsymbol{g})\right\}} \sum_{i=1}^{N} G_{i}\left(w_{i}(\boldsymbol{h}, \boldsymbol{g})\right) \\
\text { s.t. } & \sum_{i=1}^{N} w_{i}(\boldsymbol{h}, \boldsymbol{g}) \leq W
\end{aligned}
$$

where $G_{i}\left(w_{i}(\boldsymbol{h}, \boldsymbol{g})\right) \triangleq w_{i}(\boldsymbol{h}, \boldsymbol{g}) \log \left(1+h_{i} p_{i}(\boldsymbol{h}, \boldsymbol{g}) / w_{i}(\boldsymbol{h}, \boldsymbol{g})\right)$ is an increasing and concave function of $w_{i}(\boldsymbol{h}, \boldsymbol{g})$. The problem (7a) $-(7 \mathrm{~b})$ is similar to the classical water-filling power allocation problem. Thus, the optimal solution of the problem (7a)-(7b), denoted by $\left\{w_{i}^{\prime}(\boldsymbol{h}, \boldsymbol{g})\right\}$, must satisfy

$$
\left.\frac{\partial G_{i}\left(w_{i}(\boldsymbol{h}, \boldsymbol{g})\right)}{\partial w_{i}(\boldsymbol{h}, \boldsymbol{g})}\right|_{w_{i}(\boldsymbol{h}, \boldsymbol{g})=w_{i}^{\prime}(\boldsymbol{h}, \boldsymbol{g})}=\left.\frac{\partial G_{j}\left(w_{j}(\boldsymbol{h}, \boldsymbol{g})\right)}{\partial w_{j}(\boldsymbol{h}, \boldsymbol{g})}\right|_{w_{j}(\boldsymbol{h}, \boldsymbol{g})=w_{j}^{\prime}(\boldsymbol{h}, \boldsymbol{g})}, \forall i \neq j
$$


Since we have

$$
\left.\frac{\partial G_{i}\left(w_{i}(\boldsymbol{h}, \boldsymbol{g})\right)}{\partial w_{i}(\boldsymbol{h}, \boldsymbol{g})}\right|_{w_{i}\left(\boldsymbol{h}, \boldsymbol{g} \neq w_{i}^{\prime}(\boldsymbol{h}, \boldsymbol{g})\right.}=\log \left(1+\frac{h_{i} p_{i}(\boldsymbol{h}, \boldsymbol{g})}{w_{i}^{\prime}(\boldsymbol{h}, \boldsymbol{g})}\right)-\frac{h_{i} p_{i}(\boldsymbol{h}, \boldsymbol{g})}{w_{i}^{\prime}(\boldsymbol{h}, \boldsymbol{g})+h_{i} p_{i}(\boldsymbol{h}, \boldsymbol{g})}=Y\left(\frac{h_{i} p_{i}(\boldsymbol{h}, \boldsymbol{g})}{w_{i}^{\prime}(\boldsymbol{h}, \boldsymbol{g})}\right)
$$

where $Y(x) \triangleq \log (1+x)-x /(1+x)$ is a monotonically increasing function, we can obtain from (8) that

$$
\frac{h_{i} p_{i}(\boldsymbol{h}, \boldsymbol{g})}{w_{i}^{\prime}(\boldsymbol{h}, \boldsymbol{g})}=\frac{h_{j} p_{j}(\boldsymbol{h}, \boldsymbol{g})}{w_{j}^{\prime}(\boldsymbol{h}, \boldsymbol{g})}, \quad \forall i \neq j
$$

It follows from (7b) that at optimality we have $\sum_{i=1}^{N} w_{i}^{\prime}(\boldsymbol{h}, \boldsymbol{g})=W$. Furthermore, using (10), we can obtain that

$$
w_{i}^{\prime}(\boldsymbol{h}, \boldsymbol{g})=W \frac{h_{i} p_{i}(\boldsymbol{h}, \boldsymbol{g})}{\sum_{i=1}^{N} h_{i} p_{i}(\boldsymbol{h}, \boldsymbol{g})} .
$$

Substituting the optimal $w_{i}(\boldsymbol{h}, \boldsymbol{g})$ given by (11) into (6), we can equivalently rewrite (6) as

$$
\max _{\left\{p_{i}(\boldsymbol{h}, \boldsymbol{g})\right\} \in \mathcal{F}^{\prime}} \mathrm{E}\left\{W \log \left(1+\frac{\sum_{i=1}^{N} h_{i} p_{i}(\boldsymbol{h}, \boldsymbol{g})}{W}\right)\right\}
$$

where $\mathcal{F}^{\prime}$ is a feasible set specified only by a particular combination of the power constraints $\{(2),(3),(4),(5)\}$. Therefore, the optimal power allocation obtained from the problem (6) and denoted by $\left\{p_{i}^{*}(\boldsymbol{h}, \boldsymbol{g})\right\}$, can also be obtained by solving the equivalent problem (12). Then the optimal bandwidth allocation obtained from the problem (6) and denoted by $\left\{w_{i}^{*}(\boldsymbol{h}, \boldsymbol{g})\right\}$, can be found as

$$
w_{i}^{*}(\boldsymbol{h}, \boldsymbol{g})=W \frac{h_{i} p_{i}^{*}(\boldsymbol{h}, \boldsymbol{g})}{\sum_{i=1}^{N} h_{i} p_{i}^{*}(\boldsymbol{h}, \boldsymbol{g})} .
$$

\section{Optimal Power Allocation}

In this section, we study the optimal power allocation obtained from the problem (12) with $\mathcal{F}^{\prime}$ specified by different combinations of the power constraints.

\section{A. Peak transmit power with peak interference power constraints}

Consider $\mathcal{F}^{\prime}=\{$ the constraints (2) and (3) $\}$. Then the optimal value of the problem (12) can be expressed as $\mathrm{E}\left\{f_{1}(\boldsymbol{h}, \boldsymbol{g})\right\}$, where $f_{1}(\boldsymbol{h}, \boldsymbol{g})$ is given by

$$
\begin{aligned}
& f_{1}(\boldsymbol{h}, \boldsymbol{g}) \triangleq \quad \max _{\left\{p_{i}(\boldsymbol{h}, \boldsymbol{g})\right\}} W \log \left(1+\frac{\sum_{i=1}^{N} h_{i} p_{i}(\boldsymbol{h}, \boldsymbol{g})}{W}\right) \\
& \text { s.t. } p_{i}(\boldsymbol{h}, \boldsymbol{g}) \leq P_{i}^{p k}, \forall i \\
& \\
& \quad \sum_{i=1}^{N} g_{i} p_{i}(\boldsymbol{h}, \boldsymbol{g}) \leq Q^{p k} .
\end{aligned}
$$


For brevity, we drop the dependence on $\boldsymbol{h}$ and $\boldsymbol{g}$ that specifies instantaneous channel power gains. Also let $\left\{p_{i}^{*}\right\}$ denote the optimal solution of the problem (14a)-(14c). Introducing $q_{i} \triangleq g_{i} p_{i}$, the problem (14a)-114c) can be equivalently rewritten as

$$
\begin{gathered}
\max _{\left\{q_{i}\right\}} \sum_{i=1}^{N} \frac{h_{i}}{g_{i}} q_{i} \\
\text { s.t. } q_{i} \leq g_{i} P_{i}^{p k}, \forall i \\
\quad \sum_{i=1}^{N} q_{i} \leq Q^{p k} .
\end{gathered}
$$

Let $\left\{q_{i}^{*}\right\}$ denote the optimal solution of the problem (15a)-(15c) and $\left(s_{1}, s_{2}, \cdots, s_{N}\right)$ denote a permutation of the $\mathrm{SU}$ indexes such that $h_{s_{1}} / g_{s_{1}}>h_{s_{2}} / g_{s_{2}}>\cdots>h_{s_{N}} / g_{s_{N}}$. It is assumed that $h_{i} / g_{i} \neq h_{j} / g_{j}, \forall i \neq j$, since $h_{i}, g_{i}, h_{j}$, and $g_{j}$ are drawn from a continuous-valued random process. Then the following lemma is in order.

Lemma 1: There exists $k, 1 \leq k \leq N$, such that $q_{s_{i}}^{*}=g_{s_{i}} P_{s_{i}}^{p k}, \forall i, 1 \leq i \leq k-1,0<q_{s_{k}}^{*} \leq$ $g_{s_{k}} P_{s_{k}}^{p k}$, and $q_{s_{i}}^{*}=0, \forall i, k+1 \leq i \leq N$.

Proof: Let $q_{s_{j}}^{*}>0$ for some $j$ and let $l<j$ for some $l$. First we prove that $q_{s_{l}}^{*}=g_{s_{l}} P_{s_{l}}^{p k}$ by contradiction. If $q_{s_{l}}^{*}<g_{s_{l}} P_{s_{l}}^{p k}$, then we can always find $\Delta q>0$ and define a feasible solution $\left\{q_{s_{i}}^{\prime}\right\}$ of the problem (15a)-15c) $q_{s_{j}}^{\prime} \triangleq q_{s_{j}}^{*}-\Delta q, q_{s_{l}}^{\prime} \triangleq q_{s_{l}}^{*}+\Delta q, q_{s_{i}}^{\prime} \triangleq q_{s_{i}}^{*}, \forall i, i \neq j, i \neq l$ such that the objective function in (15a) achieves larger value for $\left\{q_{s_{i}}^{\prime}\right\}$ than for the optimal solution $\left\{q_{i}^{*}\right\}$, since we have

$$
\sum_{i=1}^{N} \frac{h_{s_{i}}}{g_{s_{i}}} q_{s_{i}}^{\prime}-\sum_{i=1}^{N} \frac{h_{s_{i}}}{g_{s_{i}}} q_{s_{i}}^{*}=\left(\frac{h_{s_{l}}}{g_{s_{l}}}-\frac{h_{s_{j}}}{g_{s_{j}}}\right) \Delta q>0 .
$$

Therefore, it contradicts the fact that $\left\{q_{s_{i}}^{*}\right\}$ is the optimal solution of the problem (15a)-(15c).

Let $q_{s_{j}}^{*}<g_{s_{j}} P_{s_{j}}^{p k}$ for some $j$ and let $l>j$ for some $l$. Using the result obtained above, it can be proved also by contradiction that $q_{s_{l}}^{*}=0$. This completes the proof.

Lemma 1 shows that for the optimal power allocation under the constraints (2) and (3), there exists at most one user that transmits at nonzero power and below its peak power, while any other user either does not transmit or transmits at its peak power.

Note that either the constraints (15b) or the constraint (15c) must be active at optimality. Using the structure of $\left\{q_{i}^{*}\right\}$ given in Lemma 1, $k$ can be found by Algorithm 1. 


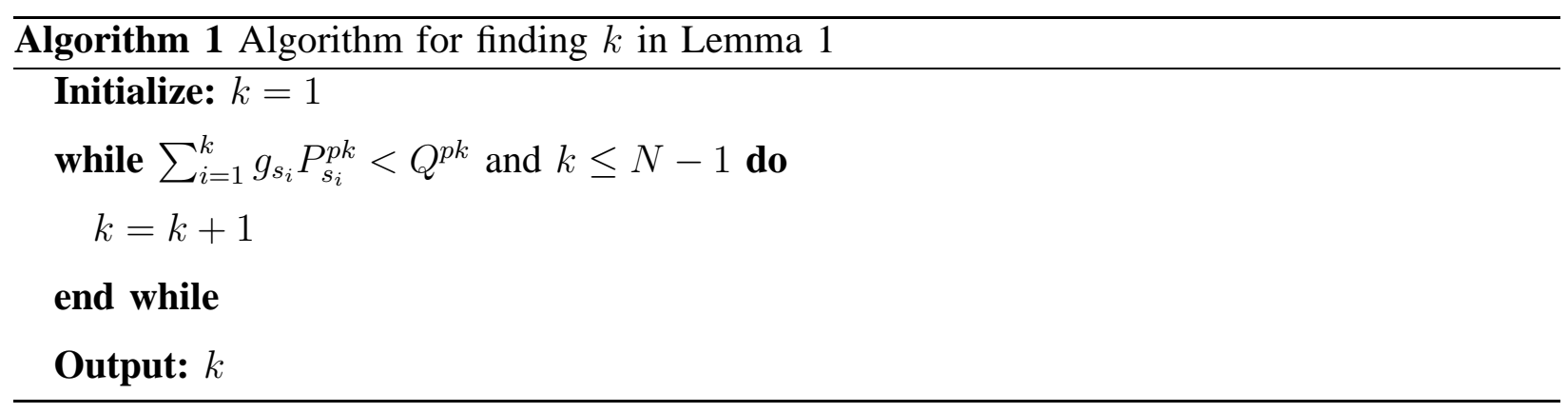

Since $p_{s_{i}}^{*}=q_{s_{i}}^{*} / g_{s_{i}}$, we obtain

$$
p_{s_{i}}^{*}= \begin{cases}P_{s_{i}}^{p k}, & 1 \leq i \leq k-1 \\ \min \left\{P_{s_{i}}^{p k},\left(Q^{p k}-\sum_{i=1}^{k-1} g_{s_{i}} P_{s_{i}}^{p k}\right) / g_{s_{i}}\right\}, & i=k \\ 0, & k+1 \leq i \leq N .\end{cases}
$$

Note that for brevity, we say in this paper that $\sum_{i=1}^{n} x_{i}=0$ if $n=0$ with a little abuse of notation.

B. Average transmit power with average interference power constraints

Consider $\mathcal{F}^{\prime}=\{$ the constraints (4) and (5) $\}$. Then the dual function of the problem (12) can be written as

$$
f_{2}\left(\left\{\lambda_{i}\right\}, \mu\right) \triangleq \mathrm{E}\left\{f_{2}^{\prime}(\boldsymbol{h}, \boldsymbol{g})\right\}+\sum_{i=1}^{N} \lambda_{i} P_{i}^{a v}+\mu Q^{a v}
$$

where $\left\{\lambda_{i} \mid 1 \leq i \leq N\right\}$ and $\mu$ are the nonnegative dual variables associated with the corresponding constraints in (4) and (5) and $f_{2}^{\prime}(\boldsymbol{h}, \boldsymbol{g})$ is given by

$$
f_{2}^{\prime}(\boldsymbol{h}, \boldsymbol{g}) \triangleq \max _{\left\{p_{i}(\boldsymbol{h}, \boldsymbol{g})\right\}} W \log \left(1+\frac{\sum_{i=1}^{N} h_{i} p_{i}(\boldsymbol{h}, \boldsymbol{g})}{W}\right)-\sum_{i=1}^{N} \gamma_{i} p_{i}(\boldsymbol{h}, \boldsymbol{g})
$$

with $\gamma_{i} \triangleq \lambda_{i}+\mu g_{i}$. Let $\left\{p_{i}^{*}\right\}$ denote the optimal solution of the problem (19), where we drop the dependence on $\boldsymbol{h}$ and $\boldsymbol{g}$ for brevity. Also let $F\left(\left\{p_{i}\right\}\right)$ denote the objective function in (19). If $p_{i}^{*}>0$ for some $i$, the following must hold

$$
\left.\frac{\partial F\left(\left\{p_{i}\right\}\right)}{\partial p_{i}}\right|_{\left\{p_{i}\right\}=\left\{p_{i}^{*}\right\}}=\frac{h_{i}}{1+\sum_{i=1}^{N} h_{i} p_{i}^{*} / W}-\gamma_{i}=0 .
$$

Then the following lemma is of interest.

Lemma 2: If $h_{i} \leq \gamma_{i}$ for some $i$, then $p_{i}^{*}=0$. 
Proof: If $p_{j}^{*}=0, \forall j$, then $p_{i}^{*}=0$. If $p_{j}^{*} \neq 0$ for some $j$, it can be seen that (20) can not be satisfied since $h_{i} \leq \gamma_{i}$. Thus, $p_{i}^{*}=0$.

If $p_{i}^{*}=0$ for some $i$, the following must hold

$$
\left.\frac{\partial F\left(\left\{p_{i}\right\}\right)}{\partial p_{i}}\right|_{\left\{p_{i}\right\}=\left\{p_{i}^{*}\right\}}=\frac{h_{i}}{1+\sum_{i=1}^{N} h_{i} p_{i}^{*} / W}-\gamma_{i} \leq 0 .
$$

Then the next lemma is in order.

Lemma 3: $p_{i}^{*}=0, \forall i$, if and only if $h_{i} \leq \gamma_{i}, \forall i$.

Proof: It can be seen from Lemma 2 that if $h_{i} \leq \gamma_{i}, \forall i$, then $p_{i}^{*}=0, \forall i$. Moreover, it can be seen from (21) that if $p_{i}^{*}=0, \forall i$, then $h_{i} \leq \gamma_{i}, \forall i$.

Let $\left(s_{1}, s_{2}, \cdots, s_{N}\right)$ denote a permutation of the SU indexes such that $h_{s_{1}} / \gamma_{s_{1}}>h_{s_{2}} / \gamma_{s_{2}}>$ $\cdots>h_{s_{N}} / \gamma_{s_{N}}$. Then we can also prove the following lemma.

Lemma 4: There exists at most one $k$ such that $p_{k}^{*}>0$. Moreover, $k=s_{1}$.

Proof: We prove it by contradiction. It can be seen from (20) that if $p_{i}^{*}>0$ and $p_{j}^{*}>0$ for some $i \neq j$, the following must hold

$$
\frac{h_{i}}{\gamma_{i}}=\frac{h_{j}}{\gamma_{j}}
$$

Since $h_{i}, \gamma_{i}, h_{j}$, and $\gamma_{j}$ are independent constants given in the problem (19), (22) can not be satisfied. Let $p_{k}^{*}>0$ and $p_{i}^{*}=0, \forall i, i \neq k$. Then it follows from (20) and (21) that the following must hold

$$
\frac{h_{k}}{\gamma_{k}} \geq \frac{h_{i}}{\gamma_{i}}, \forall i \neq k
$$

Therefore, we must have $k=s_{1}$.

Lemma 4 shows that for the optimal power allocation under the constraints (4) and (5), there exists at most one user that transmits at nonzero power, while any other user does not transmit.

Case 1: Consider the case when $h_{i} \leq \gamma_{i}, \forall i$. It follows from Lemma 3 that $p_{i}^{*}=0, \forall i$.

Case 2: Consider the case when $h_{i} \leq \gamma_{i}$ does not hold for some $i$. Using Lemma 4, let $p_{k}^{*}>0$ and $p_{i}^{*}=0, \forall i, i \neq k$. Substituting $\left\{p_{i}^{*}\right\}$ into (20), we have $p_{s_{1}}^{*}=W\left(1 / \gamma_{s_{1}}-1 / h_{s_{1}}\right)$. Therefore, we obtain

$$
p_{s_{i}}^{*}= \begin{cases}W\left(1 /\left(\lambda_{s_{1}}+\mu g_{s_{1}}\right)-1 / h_{s_{1}}\right), & i=1 \\ 0, & 2 \leq i \leq N .\end{cases}
$$




\section{Peak transmit power with average interference power constraints}

Consider $\mathcal{F}^{\prime}=\{$ the constraints (2) and (5) $\}$. Then the dual function of the problem (12) can be written as

$$
f_{3}(\mu) \triangleq \mathrm{E}\left\{f_{3}^{\prime}(\boldsymbol{h}, \boldsymbol{g})\right\}+\mu Q^{a v}
$$

where $\mu$ is the nonnegative dual variable associated with the constraint (5), and $f_{3}^{\prime}(\boldsymbol{h}, \boldsymbol{g})$ is given by

$$
\begin{aligned}
f_{3}^{\prime}(\boldsymbol{h}, \boldsymbol{g}) \triangleq \quad & \max _{\left\{p_{i}(\boldsymbol{h}, \boldsymbol{g})\right\}} W \log \left(1+\frac{\sum_{i=1}^{N} h_{i} p_{i}(\boldsymbol{h}, \boldsymbol{g})}{W}\right)-\mu \sum_{i=1}^{N} g_{i} p_{i}(\boldsymbol{h}, \boldsymbol{g}) \\
& \text { s.t. } p_{i}(\boldsymbol{h}, \boldsymbol{g}) \leq P_{i}^{p k}, \quad \forall i .
\end{aligned}
$$

Let $\left\{p_{i}^{*}\right\}$ denote the optimal solution of the problem (26a)-(26b) after dropping the dependence on $\boldsymbol{h}$ and $\boldsymbol{g}$ for brevity. The following cases are of interest.

Case 1: Consider the case when $h_{i} \leq \mu g_{i}, \forall i$. Since the problem (26a) - 26b without the constraints (26b) has the same form as the problem (19), and $p_{i}=0, \forall i$, satisfies the constraint (26b), it can be seen from Lemma 3 that $p_{i}^{*}=0, \forall i$.

Case 2: Consider the case when $h_{i} \leq \mu g_{i}$ does not hold for some $i$. The problem (26a)-(26b) is equivalent to

$$
\begin{gathered}
\max _{\left\{q_{i}\right\}} W \log \left(1+\frac{\sum_{i=1}^{N} h_{i} q_{i} / \mu g_{i}}{W}\right)-\sum_{i=1}^{N} q_{i} \\
\text { s.t. } q_{i} \leq \mu g_{i} P_{i}^{p k}, \forall i
\end{gathered}
$$

where $q_{i} \triangleq \mu g_{i} p_{i}$. Let $\left\{q_{i}^{*}\right\}$ denote the optimal solution of the problem (27a)-(27b) and $\left(s_{1}, s_{2}, \cdots, s_{N}\right)$ denote a permutation of the SU indexes such that $h_{s_{1}} / \mu g_{s_{1}}>h_{s_{2}} / \mu g_{s_{2}}>\cdots>$ $h_{s_{N}} / \mu g_{s_{N}}$. Then the following lemma is in order.

Lemma 5: There exists $k, 1 \leq k \leq N$, such that $q_{s_{i}}^{*}=g_{s_{i}} P_{s_{i}}^{p k}, \forall i, 1 \leq i \leq k-1,0<q_{s_{k}}^{*} \leq$ $g_{s_{k}} P_{s_{k}}^{p k}$, and $q_{s_{i}}^{*}=0, \forall i, k+1 \leq i \leq N$.

Proof: Consider the following intermediate problem

$$
\begin{aligned}
& \max _{\left\{q_{i}\right\}} \sum_{i=1}^{N} \frac{h_{i}}{\mu g_{i}} q_{i} \\
& \text { s.t. } q_{i} \leq \mu g_{i} P_{i}^{p k}, \forall i \\
& \quad \sum_{i=1}^{N} q_{i}=Q
\end{aligned}
$$


where $Q \triangleq \sum_{i=1}^{N} q_{i}^{*}$ and it is unknown since $\left\{q_{i}^{*}\right\}$ is unknown. Let $\left\{q_{i}^{\prime}\right\}$ denote the optimal solution of the problem (28a)-(28c). If $\left\{q_{i}^{\prime}\right\} \neq\left\{q_{i}^{*}\right\}$, we have $\sum_{i=1}^{N} h_{i} q_{i}^{\prime} / \mu g_{i} \geq \sum_{i=1}^{N} h_{i} q_{i}^{*} / \mu g_{i}$ since $\left\{q_{i}^{*}\right\}$ is a feasible solution of the problem (28a)-(28c). Then we have

$$
F\left(\left\{q_{i}^{\prime}\right\}\right)-F\left(\left\{q_{i}^{*}\right\}\right)=W \log \left(1+\frac{\sum_{i=1}^{N} h_{i} q_{i}^{\prime} / \mu g_{i}}{W}\right)-W \log \left(1+\frac{\sum_{i=1}^{N} h_{i} q_{i}^{*} / \mu g_{i}}{W}\right) \geq 0
$$

where $F\left(\left\{q_{i}\right\}\right)$ denotes the objective function in the problem (27a)-(27b). Since $\left\{q_{i}^{\prime}\right\}$ is a feasible solution of the problem (27a)-(27b), it contradicts the fact that $\left\{q_{i}^{*}\right\}$ is the optimal solution of the problem (27a) $-(27 \mathrm{~b})$. Therefore, it must be true that $\left\{q_{i}^{\prime}\right\}=\left\{q_{i}^{*}\right\}$.

It can be seen from the constraints (27b) that $\sum_{i=1}^{N} q_{i}^{\prime}=\sum_{i=1}^{N} q_{i}^{*}=Q \leq \sum_{i=1}^{N} \mu g_{i} P_{i}^{p k}$. Then the problem (28a $-(28 \mathrm{c})$ is equivalent to the following problem

$$
\begin{aligned}
& \max _{\left\{q_{i}\right\}} \sum_{i=1}^{N} \frac{h_{i}}{\mu g_{i}} q_{i} \\
& \text { s.t. } q_{i} \leq \mu g_{i} P_{i}^{p k}, \forall i \\
& \sum_{i=1}^{N} q_{i} \leq Q
\end{aligned}
$$

since the constraint ( $(30 \mathrm{c})$ is active at optimality. Therefore, the problem $(27 \mathrm{a})-(27 \mathrm{~b})$ is equivalent to the problem (30a)-(30c). Since the problem $(30 \mathrm{a})-(30 \mathrm{c})$ is similar to the problem (15a)-(15c) in Section IV-A we conclude that $\left\{q_{i}^{*}\right\}$ has the same structure as that given in Lemma 1.

The result of Lemma 5 is similar to that of Lemma 1. Specifically, it shows that for the optimal power allocation under the constraints (2) and (5), there exists at most one user that transmits at nonzero power and below its peak power, while any other user either does not transmit or transmits at its peak power.

Using Lemma 5, let $q_{s_{i}}^{*}=\mu g_{s_{i}} P_{s_{i}}^{p k}, \forall i, 1 \leq i \leq k-1,0<q_{s_{k}}^{*} \leq \mu g_{s_{i}} P_{s_{i}}^{p k}$, and $q_{s_{i}}^{*}=0, \forall i$, $k+1 \leq i \leq N$. Then we only need to find $k$ and $q_{s_{k}}^{*}$ to determine $\left\{q_{i}^{*}\right\}$.

Consider the case when $0<q_{s_{k}}^{*}<\mu g_{s_{k}} P_{s_{k}}^{p k}, 1 \leq k \leq N$. Then the following must be true

$$
\left.\frac{\partial H\left(q_{s_{k}}\right)}{\partial q_{s_{k}}}\right|_{q_{s_{k}}=q_{s_{k}}^{*}}=\frac{h_{s_{k}} / \mu g_{s_{k}}}{1+\left(\sum_{i=1, i \neq k}^{N} h_{s_{i}} q_{s_{i}}^{*} / \mu g_{s_{i}}+h_{s_{k}} q_{s_{k}}^{*} / \mu g_{s_{k}}\right) / W}-1=0
$$

where

$$
H\left(q_{s_{k}}\right) \triangleq W \log \left(1+\frac{\sum_{i=1, i \neq k}^{N} h_{s_{i}} q_{s_{i}}^{*} / \mu g_{s_{i}}+h_{s_{k}} q_{s_{k}} / \mu g_{s_{k}}}{W}\right)-\sum_{i=1, i \neq k}^{N} q_{s_{i}}^{*}-q_{s_{k}} .
$$


Substituting $\left\{q_{s_{i}}^{*}\right\}$ into (31), we obtain $q_{s_{k}}^{*}=W\left(1-\mu g_{s_{k}} / h_{s_{k}}\right)-\mu g_{s_{k}} \sum_{i=1}^{k-1} h_{s_{i}} P_{s_{i}}^{p k} / h_{s_{k}}$. Since $q_{s_{k}}^{*}$ must satisfy $0<q_{s_{k}}^{*}<\mu g_{s_{i}} P_{s_{i}}^{p k}$, it must be true that

$$
\sum_{i=1}^{k-1} h_{s_{i}} P_{s_{i}}^{p k}<W\left(\frac{h_{s_{k}}}{\mu g_{s_{k}}}-1\right)<\sum_{i=1}^{k} h_{s_{i}} P_{s_{i}}^{p k}
$$

Consider the case when $q_{s_{k}}^{*}=\mu g_{s_{k}} P_{s_{k}}^{p k}, 1 \leq k \leq N-1$. Then the following must hold

$$
\left.\frac{\partial H\left(q_{s_{k}}\right)}{\partial q_{s_{k}}}\right|_{q_{s_{k}}=q_{s_{k}}^{*}}=\frac{h_{s_{k}} / \mu g_{s_{k}}}{1+\left(\sum_{i=1, i \neq k}^{N} h_{s_{i}} q_{s_{i}}^{*} / \mu g_{s_{i}}+h_{s_{k}} q_{s_{k}}^{*} / \mu g_{s_{k}}\right) / W}-1 \geq 0
$$

and

$$
\left.\frac{\partial H\left(q_{s_{k+1}}\right)}{\partial q_{s_{k+1}}}\right|_{q_{s_{k+1}=q_{s_{k+1}}^{*}}}=\frac{h_{s_{k+1}} / \mu g_{s_{k+1}}}{1+\left(\sum_{i=1, i \neq k+1}^{N} h_{s_{i}} q_{s_{i}}^{*} / \mu g_{s_{i}}+h_{s_{k+1}} q_{s_{k+1}}^{*} / \mu g_{s_{k+1}}\right) / W}-1 \leq 0 .
$$

Substituting $\left\{q_{i}^{*}\right\}$ into (34) and (35), we obtain

$$
W\left(\frac{h_{s_{k+1}}}{\mu g_{s_{k+1}}}-1\right) \leq \sum_{i=1}^{k} h_{s_{i}} P_{s_{i}}^{p k} \leq W\left(\frac{h_{s_{k}}}{\mu g_{s_{k}}}-1\right), 1 \leq k \leq N-1 .
$$

If $q_{s_{k}}^{*}=\mu g_{s_{k}} P_{s_{k}}^{p k}, k=N$, then only (34) must be true and it follows that

$$
\sum_{i=1}^{k} h_{s_{i}} P_{s_{i}}^{p k} \leq W\left(\frac{h_{s_{k}}}{\mu g_{s_{k}}}-1\right), k=N
$$

Lemma 6: There exists only one set of values for $\left\{q_{i}^{*}\right\}$ that satisfies only one of the necessary conditions (31), (34) or (35).

Proof: It is equivalent to prove that there exists only one $k$ that satisfies only one of (33), (36) or (37). Let $L_{j} \triangleq \sum_{i=1}^{j} h_{s_{i}} P_{s_{i}}^{p k}$ and $M_{j} \triangleq W\left(h_{s_{j}} / \mu g_{s_{j}}-1\right)$ for brevity. Then it must be true that $L_{0}<L_{1}<\cdots<L_{N}, M_{1}>M_{2}>\cdots>M_{N}$ and $L_{0}<M_{1}$. It can be seen that if (37) holds, i.e., if $L_{i}<M_{i}, \forall i, 1 \leq i \leq N$, then (33) and (36) do not hold.

If (37) does not hold, then these exists such $l$ that $L_{i}<M_{i}, \forall i, 1 \leq i \leq l-1$ and $L_{i}>M_{i}$, $\forall i, 1 \leq i \leq N$. The following two cases should be considered. (i) If $L_{l-1}<M_{l}<L_{l}$, (33) holds for $k=l$. Since $L_{i}<M_{i}, \forall i, 1 \leq i \leq l-1$, (33) does not hold for $k<l$ as well. Since $M_{i}<M_{l}<L_{l} \leq L_{i-1}, \forall i, l+1 \leq i$, (33) does not hold for $k>l$. Since $L_{i}<L_{i+1}<M_{i+1}$, $\forall i, 1 \leq i \leq l-2$, (36) does not hold for $k<l-1$. Since $L_{l-1}<M_{l}$, (36) does not hold also for $k=l-1$. Moreover, since $M_{i}<L_{i}, \forall i, l \leq i$, (36) does not hold for $k>l-1$. Therefore, only (33) holds for only $k=l$. (ii) If $M_{l}<L_{l-1}<M_{l-1}$, (36) holds for $k=l-1$. Similar to the case (i), it can be proved that only (36) holds for only $k=l-1$. 


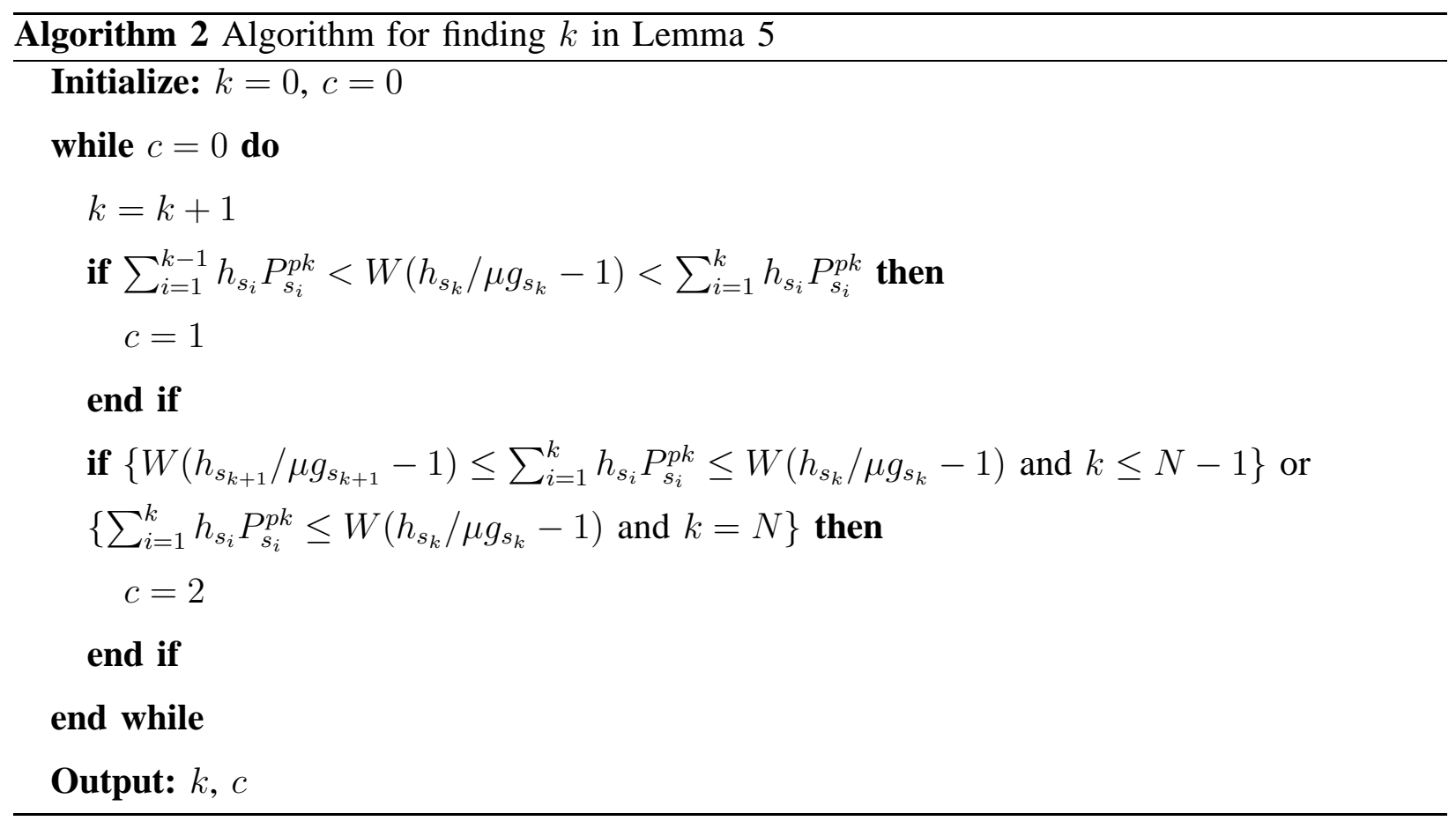

Using Lemma 6, Algorithm 2 is developed to find the unique $k$ in Lemma 5. Note that $k$ satisfies (33) and (36) or (37) if the output of Algorithm 2 is $c=1$ and $c=2$, respectively. Since $p_{s_{i}}^{*}=q_{s_{i}}^{*} / \mu g_{s_{i}}$, when $c=1$, we obtain

$$
p_{s_{i}}^{*}=\left\{\begin{array}{ll}
P_{s_{i}}^{p k}, & 1 \leq i \leq k-1 \\
W\left(1 / \mu g_{s_{k}}-1 / h_{s_{k}}\right)-\sum_{i=1}^{k-1} h_{s_{i}} P_{s_{i}}^{p k} / h_{s_{k}}, & i=k \\
0, & k+1 \leq i \leq N
\end{array} \quad, \quad 1 \leq i \leq N\right.
$$

and when $c=2$, we obtain

$$
p_{s_{i}}^{*}=\left\{\begin{array}{ll}
P_{s_{i}}^{p k}, & 1 \leq i \leq k \\
0, & k+1 \leq i \leq N
\end{array} \quad, \quad 1 \leq i \leq N .\right.
$$

D. Average transmit power with peak interference power constraints

Consider $\mathcal{F}^{\prime}=\{$ the constraints (3) and (4) $\}$. Then the dual function of the problem (12) can be written as

$$
f_{4}\left(\left\{\lambda_{i}\right\}\right) \triangleq \mathrm{E}\left\{f_{4}^{\prime}(\boldsymbol{h}, \boldsymbol{g})\right\}+\sum_{i=1}^{N} \lambda_{i} P_{i}^{a v}
$$


where $\left\{\lambda_{i} \mid 1 \leq i \leq N\right\}$ are the nonnegative dual variables associated with the corresponding constraints (4) and $f_{4}^{\prime}(\boldsymbol{h}, \boldsymbol{g})$ is given by

$$
\begin{aligned}
f_{4}^{\prime}(\boldsymbol{h}, \boldsymbol{g}) \triangleq \quad & \max _{\left\{p_{i}(\boldsymbol{h}, \boldsymbol{g})\right\}} W \log \left(1+\frac{\sum_{i=1}^{N} h_{i} p_{i}(\boldsymbol{h}, \boldsymbol{g})}{W}\right)-\sum_{i=1}^{N} \lambda_{i} p_{i}(\boldsymbol{h}, \boldsymbol{g}) \\
\text { s.t. } & \sum_{i=1}^{N} g_{i} p_{i}(\boldsymbol{h}, \boldsymbol{g}) \leq Q^{p k} .
\end{aligned}
$$

Let $\left\{p_{i}^{*}\right\}$ denote the optimal solution of the problem (41a)-(41b) where the dependence on $\boldsymbol{h}$ and $\boldsymbol{g}$ is dropped for brevity. The following three cases are of interest.

Case 1: Consider the case when $h_{i} \leq \lambda_{i}, \forall i$. Similar to Case 1 in Section IV-C, it can be seen from Lemma 3 that $p_{i}^{*}=0, \forall i$.

Case 2: Consider the case when $h_{i} \leq \lambda_{i}$ does not hold for some $i$ and the constraint (41b) is inactive at optimality. Let $\left(s_{1}, s_{2}, \cdots, s_{N}\right)$ denote a permutation of the SU indexes such that $h_{s_{1}} / \lambda_{s_{1}}>h_{s_{2}} / \lambda_{s_{2}}>\cdots>h_{s_{N}} / \lambda_{s_{N}}$. Since the problem (41a)-(41b) without the constraint (41b) has the same form as the problem (19), it can be seen from (24) that $p_{s_{1}}^{*}=W\left(1 / \lambda_{s_{1}}-1 / h_{s_{1}}\right)$ and $p_{s_{i}}^{*}=0, \forall i, 2 \leq i \leq N$, if it satisfies the constraint (41b), i.e., $\sum_{i=1}^{N} g_{s_{i}} p_{s_{i}}^{*}=g_{s_{1}} W\left(1 / \lambda_{s_{1}}-\right.$ $\left.1 / h_{s_{1}}\right)<Q^{p k}$.

Case 3: Consider the case when $h_{i} \leq \lambda_{i}$ does not hold for some $i$ and the constraint (41b) is active at optimality, i.e., $g_{s_{1}} W\left(1 / \lambda_{s_{1}}-1 / h_{s_{1}}\right) \geq Q^{p k}$. The dual function of the problem (41a)(41b) can be written as $f_{4}^{\prime \prime}(\mu) \triangleq f_{4}^{\prime \prime \prime}+\mu Q^{p k}$, where $\mu$ is the nonnegative dual variable associated with the constraint (41b), and $f_{4}^{\prime \prime \prime}$ is given by

$$
f_{4}^{\prime \prime \prime} \triangleq \max _{\left\{p_{i}\right\}} W \log \left(1+\frac{\sum_{i=1}^{N} h_{i} p_{i}}{W}\right)-\sum_{i=1}^{N} \lambda_{i} p_{i}-\mu \sum_{i=1}^{N} g_{i} p_{i} .
$$

Let $\mu^{*}$ denote the optimal dual variable. Also let $F\left(\left\{p_{i}\right\}\right)$ denote the objective function in the problem (42). If $p_{i}^{*}>0$ for some $i$, the following must hold

$$
\left.\frac{\partial F\left(\left\{p_{i}\right\}\right)}{\partial p_{i}}\right|_{\left\{p_{i}\right\}=\left\{p_{i}^{*}\right\}}=\frac{h_{i}}{1+\sum_{i=1}^{N} h_{i} p_{i}^{*} / W}-\lambda_{i}-\mu^{*} g_{i}=0 .
$$

If $p_{i}^{*}=0$ for some $i$, the following must hold

$$
\left.\frac{\partial F\left(\left\{p_{i}\right\}\right)}{\partial p_{i}}\right|_{\left\{p_{i}\right\}=\left\{p_{i}^{*}\right\}}=\frac{h_{i}}{1+\sum_{i=1}^{N} h_{i} p_{i}^{*} / W}-\lambda_{i}-\mu^{*} g_{i} \leq 0 .
$$

Note that since the problem (41a)-(41b) is convex, the necessary conditions (43) and (44) for the optimal solution $\left\{p_{i}^{*}\right\}$ are also sufficient conditions. 
Lemma 7: There exists at most two $j \neq k$ such that $p_{j}^{*}>0$ and $p_{k}^{*}>0$.

Proof: We prove it by contradiction. It can be seen from (43) that if $p_{i}^{*}>0, p_{j}^{*}>0$, and $p_{k}^{*}>0$ for some $i \neq j, j \neq k, i \neq k$, the following must hold

$$
\frac{h_{i}}{\lambda_{i}+\mu^{*} g_{i}}=\frac{h_{j}}{\lambda_{j}+\mu^{*} g_{j}}=\frac{h_{k}}{\lambda_{k}+\mu^{*} g_{k}} \text {. }
$$

Since $h_{i}, \lambda_{i}, g_{i}, h_{j}, \lambda_{j}, g_{j}, h_{k}, \lambda_{k}$, and $g_{k}$ are independent constants given in the problem (41a)-(41b), and only $\mu^{*}$ is a variable, (45) can not be satisfied.

Lemma 7 shows that for the optimal power allocation under the constraints (3) and (4), there exists at most two users that transmit at nonzero power, while any other user does not transmit.

Then Case 3 can be further divided into the following two subcases.

Case 3.1: Consider the subcase when $p_{k}^{*}>0$ and $p_{i}^{*}=0, \forall i \neq k$. Since the constraint (41b) is active at optimality, i.e., $\sum_{i=1}^{N} g_{i} p_{i}^{*}=g_{k} p_{k}^{*}=Q^{p k}$, we obtain that $p_{k}^{*}=Q^{p k} / g_{k}$. Then substituting $\left\{p_{i}^{*}\right\}$ into (43) we have

$$
\mu^{*}=\frac{1}{g_{k} / h_{k}+Q^{p k} / W}-\frac{\lambda_{k}}{g_{k}} .
$$

Note that $\mu^{*}$ given in (46) must satisfy $\mu^{*} \geq 0$. Substituting $\left\{p_{i}^{*}\right\}$ into (44), we can see that $\mu^{*}$ given in (46) also must satisfy

$$
\mu^{*} \geq \frac{h_{i} / g_{i}}{1+h_{k} Q^{p k} / g_{k} W}-\frac{\lambda_{i}}{g_{i}}, \quad \forall i, i \neq k .
$$

Then Algorithm 3 can be used to find $k$. Note that $\left\{p_{i}^{*}\right\}$ does not exist in Case 3.1 if the output

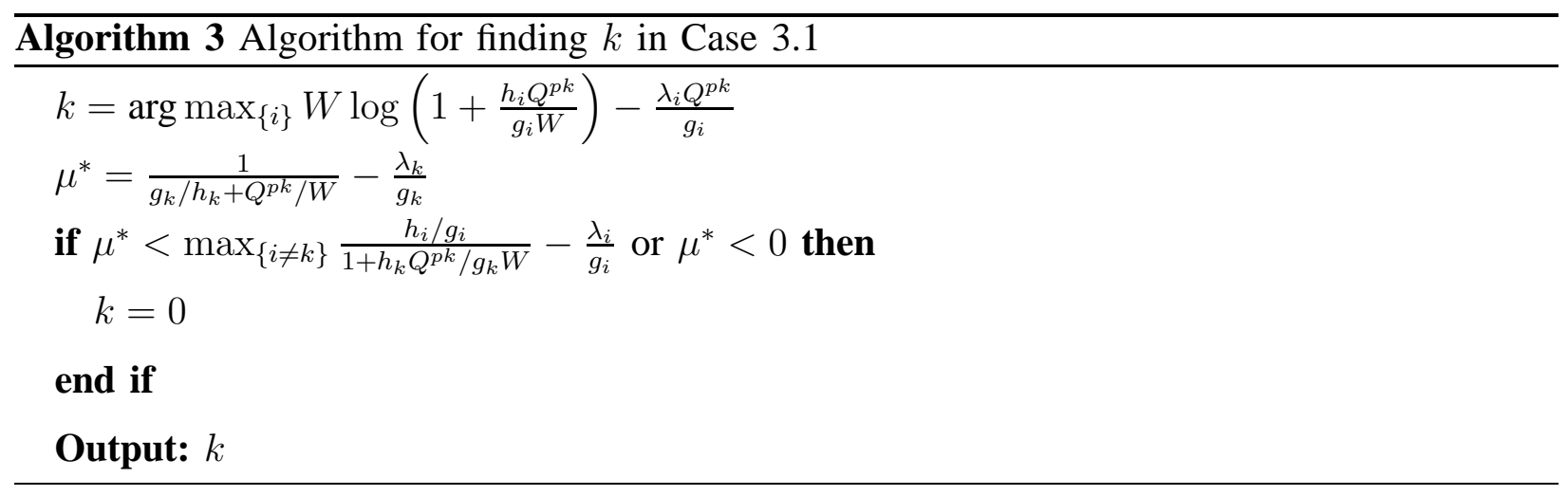

of Algorithm 3 is $k=0$.

Case 3.2: Consider the subcase when $p_{j}^{*}>0, p_{k}^{*}>0, j \neq k$ and $p_{i}^{*}=0, \forall i, i \neq j, i \neq k$. It follows from (43) that

$$
\frac{h_{j}}{\lambda_{j}+\mu^{*} g_{j}}=\frac{h_{k}}{\lambda_{k}+\mu^{*} g_{k}} .
$$


Therefore, we obtain that

$$
\mu^{*}=\frac{\lambda_{j} / h_{j}-\lambda_{k} / h_{k}}{g_{k} / h_{k}-g_{j} / h_{j}} .
$$

Note that $\mu^{*}$ given in (49) must satisfy $\mu^{*} \geq 0$. Using (43) and the fact that the constraint (41b) is active at optimality, we have

$$
\left\{\begin{array}{l}
h_{j} p_{j}^{*}+h_{k} p_{k}^{*}=W h_{j} /\left(\lambda_{j}+\mu^{*} g_{j}\right)-W \\
g_{j} p_{j}^{*}+g_{k} p_{k}^{*}=Q^{p k} .
\end{array}\right.
$$

Solving the system of equation (50), we obtain

$$
p_{j}^{*}=\frac{Q^{p k} / g_{k}-a / h_{k}}{g_{j} / g_{k}-h_{j} / h_{k}}, \quad p_{k}^{*}=\frac{a / h_{j}-Q^{p k} / g_{j}}{h_{k} / h_{j}-g_{k} / g_{j}}
$$

where $a \triangleq W h_{j} /\left(\lambda_{j}+\mu^{*} g_{j}\right)-W$. Note that $p_{j}^{*}$ and $p_{k}^{*}$ given in (51) must satisfy $p_{j}^{*}>0$ and $p_{k}^{*}>0$. Substituting $\left\{p_{i}^{*}\right\}$ and $\mu^{*}$ into (44), we can see that $j$ and $k$ must satisfy

$$
\frac{\lambda_{j} / h_{j}-\lambda_{k} / h_{k}}{g_{k} / h_{k}-g_{j} / h_{j}} \geq \frac{\lambda_{j} / h_{j}-\lambda_{i} / h_{i}}{g_{i} / h_{i}-g_{j} / h_{j}}, \quad \forall i, i \neq j, i \neq k .
$$

Then Algorithm 4 can be used to find $j$ and $k$. Note that $\left\{p_{i}^{*}\right\}$ does not exist if the output of Algorithm 4 is $j=0$ and $k=0$.

\section{E. Combinations of more than two power constraints}

Consider $\mathcal{F}^{\prime}=\{$ the constraints (2), (4), and (5) $\}$ or $\mathcal{F}^{\prime}=\{$ the constraints (3), (4), and (5) $\}$. It can be shown that the corresponding dual functions of the problem (12) under these two combinations of the power constraints have the same form as those in Subsections IV-C and IV-D respectively. Therefore, optimal solutions can be found similarly therein and, thus, are omitted here.

Consider $\mathcal{F}^{\prime}=\{$ the constraints (2), (3), and (4) $\}$ or $\mathcal{F}^{\prime}=\{$ the constraints (2), (3), and (5) $\}$ or $\mathcal{F}^{\prime}=\{$ the constraints (2), (3), (4), and (5) $\}$. It can be shown that the corresponding dual functions of the problem (12) under the first two combinations of the power constraints have the same form as that under the third combination. Therefore, we only focus on the case $\mathcal{F}^{\prime}=\{$ the constraints(2), (3), (4), and (5) $\}$. Then the dual function of the problem (12) can be written as

$$
f_{5}\left(\left\{\lambda_{i}\right\}, \mu\right) \triangleq \mathrm{E}\left\{f_{5}^{\prime}(\boldsymbol{h}, \boldsymbol{g})\right\}+\sum_{i=1}^{N} \lambda_{i} P_{i}^{a v}+\mu Q^{a v}
$$




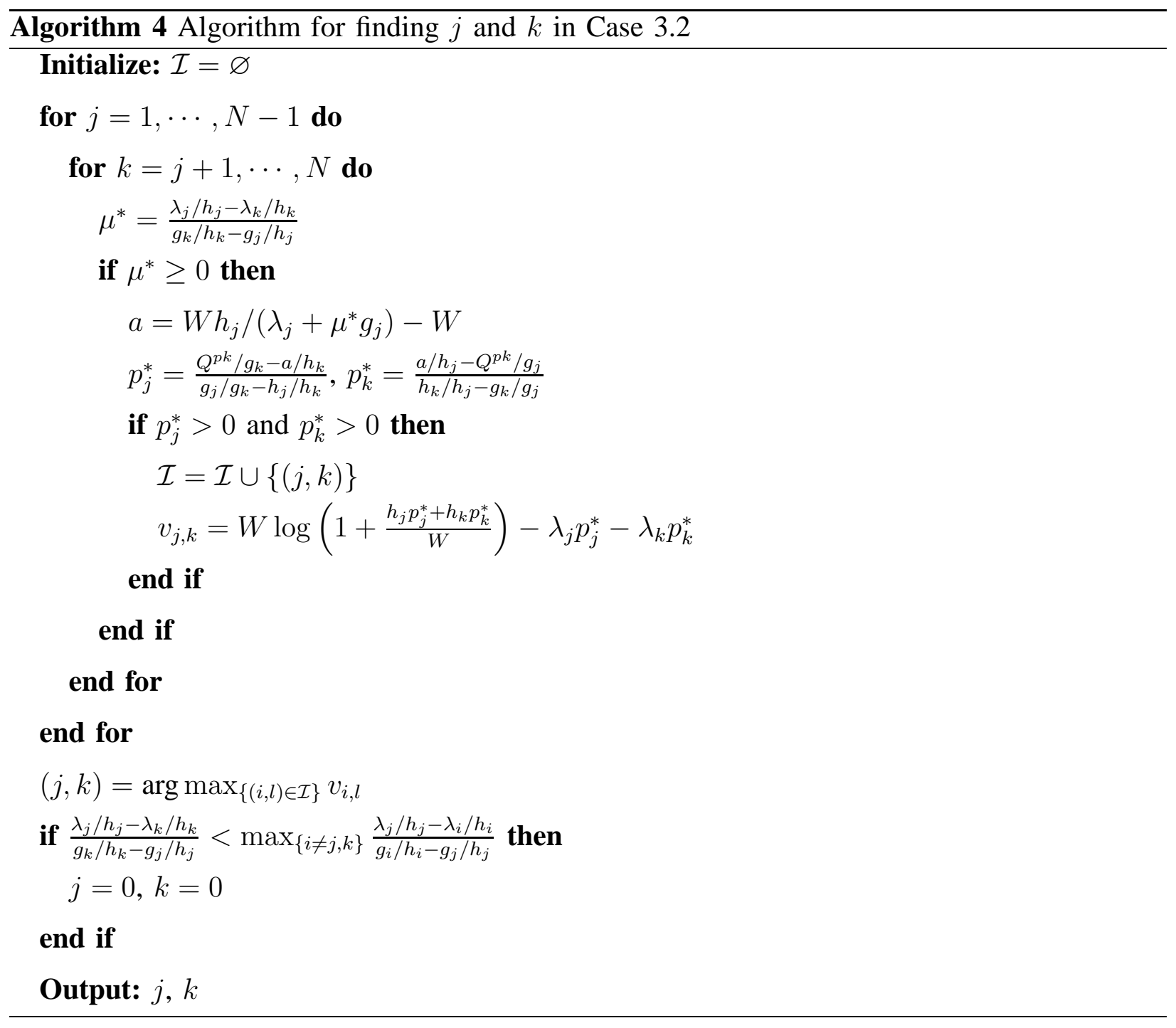

where $\left\{\lambda_{i} \mid 1 \leq i \leq N\right\}$ and $\mu$ are the nonnegative dual variables associated with the corresponding constraints in (4) and (5) and $f_{5}^{\prime}(\boldsymbol{h}, \boldsymbol{g})$ is given by

$$
\begin{aligned}
f_{5}^{\prime}(\boldsymbol{h}, \boldsymbol{g}) \triangleq & \max _{\left\{p_{i}(\boldsymbol{h}, \boldsymbol{g})\right\}} W \log \left(1+\frac{\sum_{i=1}^{N} h_{i} p_{i}(\boldsymbol{h}, \boldsymbol{g})}{W}\right)-\sum_{i=1}^{N} \lambda_{i} p_{i}(\boldsymbol{h}, \boldsymbol{g})-\mu \sum_{i=1}^{N} g_{i} p_{i}(\boldsymbol{h}, \boldsymbol{g}) \\
& \text { s.t. } \sum_{i=1}^{N} g_{i} p_{i}(\boldsymbol{h}, \boldsymbol{g}) \leq Q^{p k} \\
& p_{i}(\boldsymbol{h}, \boldsymbol{g}) \leq P_{i}^{p k}, \forall i .
\end{aligned}
$$

Let $\left\{p_{i}^{*}\right\}$ denote the optimal solution of the problem (54a)-(54c) where the dependence on $\boldsymbol{h}$ and $\boldsymbol{g}$ is dropped for brevity. The following cases are of interest. 
Case 1: Consider the case when $h_{i} \leq \lambda_{i}+\mu g_{i}, \forall i$. Similar to Case 1 in Subsections $I V-C$ and IV-D, it can be seen from Lemma 3 that $p_{i}^{*}=0, \forall i$.

Case 2: Consider the case when $h_{i} \leq \lambda_{i}+\mu g_{i}$ does not hold for some $i$ and the constraint (54b) is inactive at optimality. Since the problem (54a)-(54c) without the constraint (54b) has the same form as the problem (26a)-(26b), $\left\{p_{i}^{*}\right\}$ can be found using Algorithm 2 and (38) or (39) if it satisfies the constraint (54b).

Case 3: Consider the case when $h_{i} \leq \lambda_{i}+\mu g_{i}$ does not hold for some $i$ and the constraint (54b) is active at optimality. The dual function of the problem (54a)-(54c) can be written as $f_{5}^{\prime \prime}(\beta) \triangleq f_{5}^{\prime \prime \prime}+\beta Q^{p k}$, where $\beta$ is the nonnegative dual variable associated with the constraint (54b) and $f_{5}^{\prime \prime \prime}$ is given by

$$
\begin{aligned}
f_{5}^{\prime \prime \prime} \triangleq \quad & \max _{\left\{p_{i}\right\}} W \log \left(1+\frac{\sum_{i=1}^{N} h_{i} p_{i}}{W}\right)-\sum_{i=1}^{N} \gamma_{i} p_{i}-\beta \sum_{i=1}^{N} g_{i} p_{i} \\
& \text { s.t. } p_{i} \leq P_{i}^{p k}, \forall i .
\end{aligned}
$$

where $\gamma_{i} \triangleq \lambda_{i}+\mu g_{i}$. Let $\beta^{*}$ denote the optimal dual variable and $F\left(\left\{p_{i}\right\}\right)$ stands for the objective function in the problem (55a). If $P_{i}^{p k}>p_{i}^{*}>0$ for some $i$, the following must hold

$$
\left.\frac{\partial F\left(\left\{p_{i}\right\}\right)}{\partial p_{i}}\right|_{\left\{p_{i}\right\}=\left\{p_{i}^{*}\right\}}=\frac{h_{i}}{1+\sum_{i=1}^{N} h_{i} p_{i}^{*} / W}-\gamma_{i}-\beta^{*} g_{i}=0 .
$$

If $p_{i}^{*}=P_{i}^{p k}$ for some $i$, the following must hold

$$
\left.\frac{\partial F\left(\left\{p_{i}\right\}\right)}{\partial p_{i}}\right|_{\left\{p_{i}\right\}=\left\{p_{i}^{*}\right\}}=\frac{h_{i}}{1+\sum_{i=1}^{N} h_{i} p_{i}^{*} / W}-\gamma_{i}-\beta^{*} g_{i} \geq 0 .
$$

Moreover, if $p_{i}^{*}=0$ for some $i$, the following must hold

$$
\left.\frac{\partial F\left(\left\{p_{i}\right\}\right)}{\partial p_{i}}\right|_{\left\{p_{i}\right\}=\left\{p_{i}^{*}\right\}}=\frac{h_{i}}{1+\sum_{i=1}^{N} h_{i} p_{i}^{*} / W}-\gamma_{i}-\beta^{*} g_{i} \leq 0 .
$$

Note that since the problem (54a)-(54c) is convex, the necessary conditions (56), (57) and (58) for the optimal solution $\left\{p_{i}^{*}\right\}$ are also sufficient conditions.

Lemma 8: There exists at most two $j$ and $k, j \neq k$ such that $P_{j}^{p k}>p_{j}^{*}>0$ and $P_{k}^{p k}>p_{k}^{*}>0$.

Proof: We prove it by contradiction. It can be seen from (56) that if $P_{i}^{p k}>p_{i}^{*}>0, P_{j}^{p k}>$ $p_{j}^{*}>0$, and $P_{k}^{p k}>p_{k}^{*}>0$ for some $i \neq j, j \neq k, i \neq k$, the following must be true

$$
\frac{h_{i}}{\gamma_{i}+\beta^{*} g_{i}}=\frac{h_{j}}{\gamma_{j}+\beta^{*} g_{j}}=\frac{h_{k}}{\gamma_{k}+\beta^{*} g_{k}} \text {. }
$$


Since $h_{i}, \gamma_{i}, g_{i}, h_{j}, \gamma_{j}, g_{j}, h_{k}, \gamma_{k}$, and $g_{k}$ are independent constants given in the problem (54a)-(54c), and only $\beta^{*}$ is a variable, (59) can not be satisfied.

Lemma 8 shows that for the optimal power allocation under the constraints (2), (3), (4) and (5), there exists at most two user that transmit at nonzero power and below their peak power, while any other user either does not transmit or transmits at its peak power.

Then Case 3 can be further divided into the following two subcases.

Case 3.1: Consider the subcase when $P_{k}^{p k}>p_{k}^{*}>0$ and $p_{i}^{*} \in\left\{P_{i}^{p k}, 0\right\}, \forall i \neq k$. Let $\mathcal{N}_{1}$ and $\mathcal{N}_{0}$ denote the sets of SU indexes such that $p_{i}^{*}=P_{i}^{p k}, \forall i \in \mathcal{N}_{1}$ and $p_{i}^{*}=0, \forall i \in \mathcal{N}_{0}$. Since the constraint (54b) is active at optimality, i.e., $\sum_{i=1}^{N} g_{i} p_{i}^{*}=g_{k} p_{k}^{*}+\sum_{i \in \mathcal{N}_{1}} g_{i} P_{i}^{p k}=Q^{p k}$, we obtain $p_{k}^{*}=\left(Q^{p k}-\sum_{i \in \mathcal{N}_{1}} g_{i} P_{i}^{p k}\right) / g_{k}$. Note that $p_{k}^{*}$ given here must satisfy $P_{k}^{p k}>p_{k}^{*}>0$. Then substituting $\left\{p_{i}^{*}\right\}$ into (56) we obtain

$$
\beta^{*}=\frac{h_{k} / g_{k}}{1+\left(h_{k}\left(Q^{p k}-\sum_{i \in \mathcal{N}_{1}} g_{i} P_{i}^{p k}\right) / g_{k}+\sum_{i \in \mathcal{N}_{1}} h_{i} P_{i}^{p k}\right) / W}-\frac{\gamma_{k}}{g_{k}} .
$$

Note that $\beta^{*}$ given by (60) must satisfy $\beta^{*} \geq 0$. Substituting $\left\{p_{i}^{*}\right\}$ into (57) we can see that $\beta^{*}$ given by (60) must satisfy

$$
\beta^{*} \leq \frac{h_{i} / g_{i}}{1+\left(h_{k}\left(Q^{p k}-\sum_{i \in \mathcal{N}_{1}} g_{i} P_{i}^{p k}\right) / g_{k}+\sum_{i \in \mathcal{N}_{1}} h_{i} P_{i}^{p k}\right) / W}-\frac{\gamma_{i}}{g_{i}}, \forall i \in \mathcal{N}_{1} .
$$

Substituting $\left\{p_{i}^{*}\right\}$ into (58), we can see that $\beta^{*}$ given in (60) also must satisfy

$$
\beta^{*} \geq \frac{h_{i} / g_{i}}{1+\left(h_{k}\left(Q^{p k}-\sum_{i \in \mathcal{N}_{1}} g_{i} P_{i}^{p k}\right) / g_{k}+\sum_{i \in \mathcal{N}_{1}} h_{i} P_{i}^{p k}\right) / W}-\frac{\gamma_{i}}{g_{i}}, \forall i \in \mathcal{N}_{0} .
$$

Let $\mathcal{S}_{i}^{(1)}, \mathcal{S}_{i}^{(2)}, \cdots, \mathcal{S}_{i}^{\left(2^{N-1}\right)}$ denote all the subsets of the set $\mathcal{N} \backslash\{i\}$ where $\backslash$ denotes the set difference operator. Then Algorithm 5 can be used to find $k, \mathcal{N}_{1}$, and $\mathcal{N}_{0}$. Note that $\left\{p_{i}^{*}\right\}$ does not exist if the output of Algorithm 5 is $k=0$.

Case 3.2: Consider the subcase when $P_{j}^{p k}>p_{j}^{*}>0, P_{k}^{p k}>p_{k}^{*}>0$ and $p_{i}^{*} \in\left\{P_{i}^{p k}, 0\right\}$, $\forall i \neq j, k$. Let $\mathcal{N}_{1}$ and $\mathcal{N}_{0}$ denote the sets of SU indexes such that $p_{i}^{*}=P_{i}^{p k}, \forall i \in \mathcal{N}_{1}$ and $p_{i}^{*}=0, \forall i \in \mathcal{N}_{0}$, respectively. It follows from (56) that

$$
\frac{h_{j}}{\gamma_{j}+\beta^{*} g_{j}}=\frac{h_{k}}{\gamma_{k}+\beta^{*} g_{k}}
$$

Therefore, we obtain that

$$
\beta^{*}=\frac{\gamma_{j} / h_{j}-\gamma_{k} / h_{k}}{g_{k} / h_{k}-g_{j} / h_{j}} .
$$




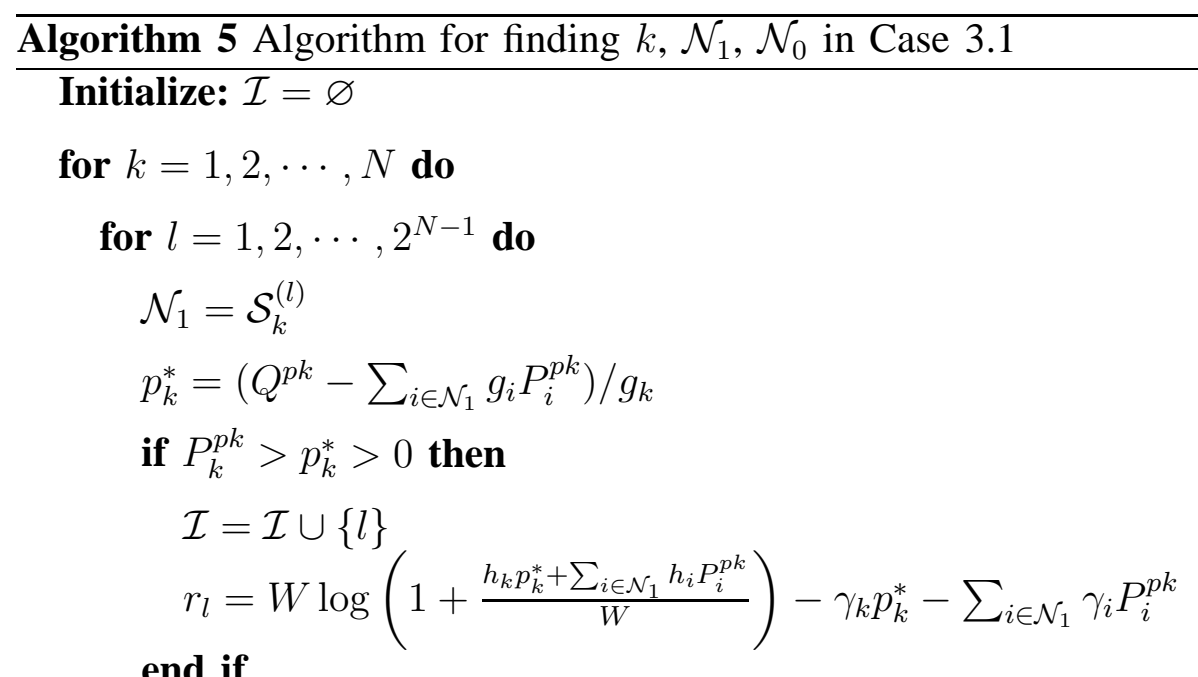

\section{end for}

$$
\begin{aligned}
& v_{k}=\max _{\{i \in \mathcal{I}\}} r_{i}, t=\arg \max _{\{i \in \mathcal{I}\}} r_{i} \\
& \mathcal{S}_{k}^{*}=\mathcal{S}_{k}^{(t)} \\
& \mathcal{I}=\varnothing
\end{aligned}
$$

\section{end for}

$$
\begin{aligned}
& k=\arg \max _{\{i\}} v_{i} \\
& \mathcal{N}_{1}=\mathcal{S}_{k}^{*} \\
& \mathcal{N}_{0}=\mathcal{N} \backslash \mathcal{N}_{1} \backslash\{k\} \\
& \beta^{*}=\frac{h_{k} / g_{k}}{1+\left(h_{k}\left(Q^{p k}-\sum_{i \in \mathcal{N}_{1}} g_{i} P_{i}^{p k}\right) / g_{k}+\sum_{i \in \mathcal{N}_{1}} h_{i} P_{i}^{p k}\right) / W}-\frac{\gamma_{k}}{g_{k}} \\
& \text { if } \beta^{*}<0 \text { or } \beta^{*}>\frac{h_{i} / g_{i}}{1+\left(h_{k}\left(Q^{p k}-\sum_{i \in \mathcal{N}_{1}} g_{i} P_{i}^{p k}\right) / g_{k}+\sum_{i \in \mathcal{N}_{1}} h_{i} P_{i}^{p k}\right) / W}-\frac{\gamma_{i}}{g_{i}}, \exists i \in \mathcal{N}_{1} \\
& \text { or } \beta^{*}<\frac{h_{i} / g_{i}}{1+\left(h_{k}\left(Q^{p k}-\sum_{i \in \mathcal{N}_{1}} g_{i} P_{i}^{p k}\right) / g_{k}+\sum_{i \in \mathcal{N}_{1}} h_{i} P_{i}^{p k}\right) / W}-\frac{\gamma_{i}}{g_{i}}, \exists i \in \mathcal{N}_{0} \text { then } \\
& \quad k=0
\end{aligned}
$$

\section{end if}

Output: $k, \mathcal{N}_{1}, \mathcal{N}_{0}$

Note that $\beta^{*}$ given in (64) must satisfy $\beta^{*} \geq 0$. Following (56) and the fact that the constraint (54b) is active at optimality, we have

$$
\left\{\begin{array}{l}
h_{j} p_{j}^{*}+h_{k} p_{k}^{*}=W h_{j} /\left(\gamma_{j}+\beta^{*} g_{j}\right)-W-\sum_{i \in \mathcal{N}_{1}} h_{i} P_{i}^{p k} \\
g_{j} p_{j}^{*}+g_{k} p_{k}^{*}=Q^{p k}-\sum_{i \in \mathcal{N}_{1}} g_{i} P_{i}^{p k}
\end{array}\right.
$$


Solving the system of equation (65), we obtain

$$
p_{j}^{*}=\frac{a / g_{k}-b / h_{k}}{g_{j} / g_{k}-h_{j} / h_{k}}, \quad p_{k}^{*}=\frac{b / h_{j}-a / g_{j}}{h_{k} / h_{j}-g_{k} / g_{j}}
$$

where $a \triangleq Q^{p k}-\sum_{i \in \mathcal{N}_{1}} g_{i} P_{i}^{p k}$ and $b \triangleq W h_{j} /\left(\gamma_{j}+\beta^{*} g_{j}\right)-W-\sum_{i \in \mathcal{N}_{1}} h_{i} P_{i}^{p k}$. Note that $p_{j}^{*}$ and $p_{k}^{*}$ given in (66) must satisfy $P_{j}^{p k}>p_{j}^{*}>0$ and $P_{k}^{p k}>p_{k}^{*}>0$. Substituting $\left\{p_{i}^{*}\right\}$ and $\beta^{*}$ given by (64) into (57), we obtain

$$
\frac{\gamma_{j} / h_{j}-\gamma_{k} / h_{k}}{g_{k} / h_{k}-g_{j} / h_{j}} \leq \frac{\gamma_{j} / h_{j}-\gamma_{i} / h_{i}}{g_{i} / h_{i}-g_{j} / h_{j}}, \quad \forall i \in \mathcal{N}_{1} .
$$

Moreover, substituting $\left\{p_{i}^{*}\right\}$ and $\beta^{*}$ given by (64) into (58), we also obtain

$$
\frac{\gamma_{j} / h_{j}-\gamma_{k} / h_{k}}{g_{k} / h_{k}-g_{j} / h_{j}} \geq \frac{\gamma_{j} / h_{j}-\gamma_{i} / h_{i}}{g_{i} / h_{i}-g_{j} / h_{j}}, \quad \forall i \in \mathcal{N}_{0} .
$$

Let $\mathcal{S}_{i, j}^{(1)}, \mathcal{S}_{i, j}^{(2)}, \cdots, \mathcal{S}_{i, j}^{\left(2^{N-2}\right)}$ denote all the subsets of the set $\mathcal{N} \backslash\{i, j\}$. Then Algorithm 6 can be used to find $j, k, \mathcal{N}_{1}$, and $\mathcal{N}_{0}$. Note that $\left\{p_{i}^{*}\right\}$ does not exist if the output of Algorithm 6 is $j=0$ and $k=0$.

\section{Simulation Results}

Consider a cognitive radio network which consists of one PU and four SUs. For simplicity, we assume that only Rayleigh fading is present in all links. The variance of the channel power gain is set to $\sigma^{2}=1$. We also set $W=1, P_{i}^{p k}=10, \forall i, P_{i}^{a v}=10, \forall i, Q^{p k}=1$, and $Q^{a v}=1$ as default values if no other values are specified otherwise. The AWGN with unit PSD is assumed. We use 1000 randomly generated channel power gains for $\boldsymbol{h}$ and $\boldsymbol{g}$ in our simulations. The results are compared under the following five combinations of the power constraints: the PTP with PIP constraints (PTP+PIP), the PTP with AIP constraints (PTP+AIP), the ATP with PIP constraints (ATP+PIP), the ATP with AIP constraints (ATP+AIP), the PTP and ATP with PIP and AIP constraints (PTP+ATP+PIP+AIP).

First, we aim at showing by Fig. 1 that the information-theoretic limit for the sum ergodic capacity is indeed significantly higher when bandwidth is allocated optimally as compared to the case when it is allocated equally among SUs. In this figure, OBPA stands for optimal bandwidth and power allocation, while EBPA stands for equal bandwidth and power allocation. The case of PTP+PIP is only depicted in Fig. 1, but the conclusion about the superiority of optimal bandwidth and power allocation holds true for other combinations of power constraints. Then 


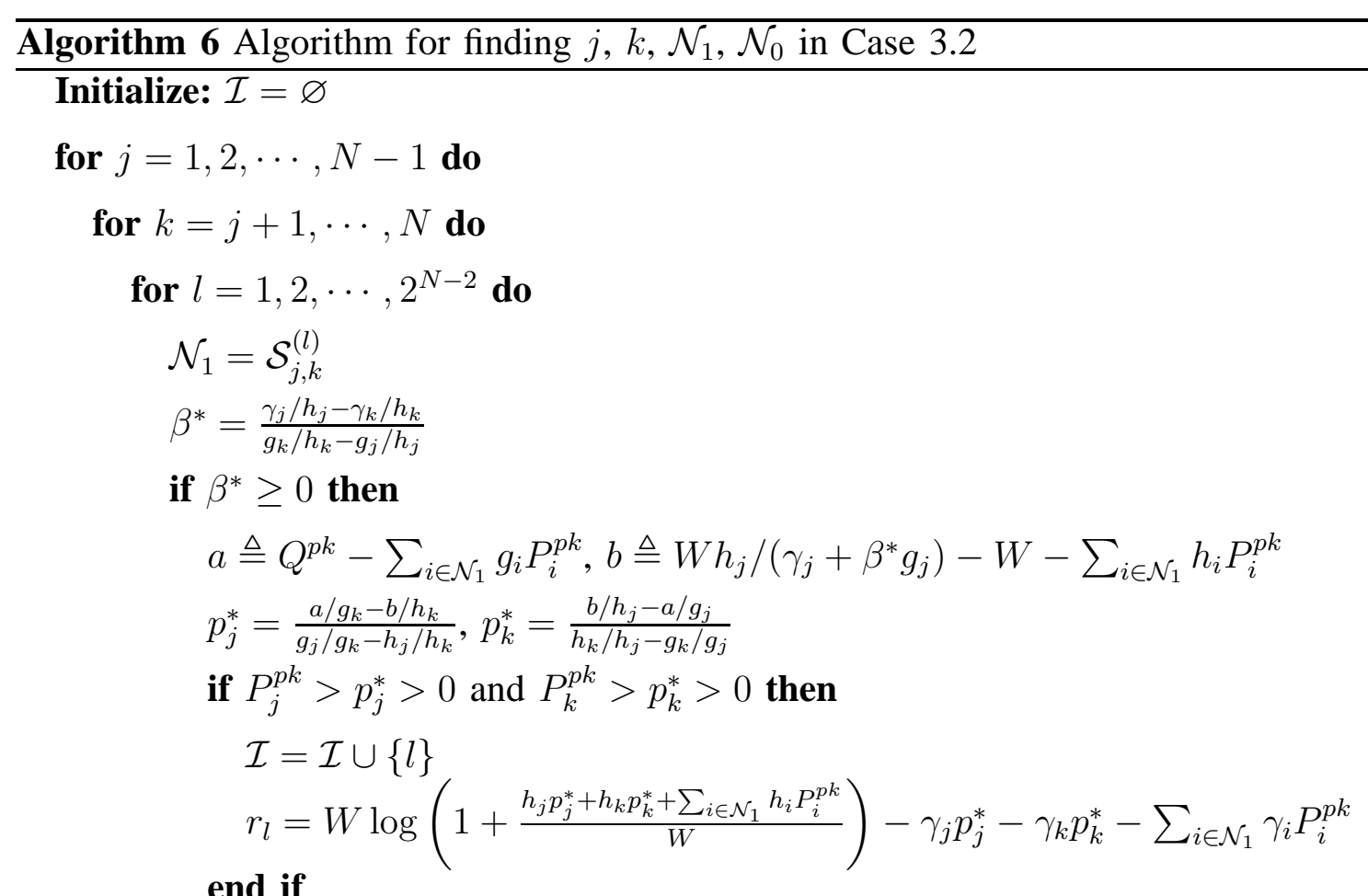

\section{end if}

\section{end for}

$$
\begin{aligned}
& v_{j, k}=\max _{\{i \in \mathcal{I}\}} r_{i}, t=\arg \max _{\{i \in \mathcal{I}\}} r_{i} \\
& \mathcal{S}_{j, k}^{*}=\mathcal{S}_{j, k}^{(t)} \\
& \mathcal{I}=\varnothing
\end{aligned}
$$

\section{end for}

\section{end for}

$$
\begin{aligned}
& (j, k)=\arg \max _{\{(i, l)\}} v_{i, l} \\
& \mathcal{N}_{1}=\mathcal{S}_{j, k}^{*} \\
& \mathcal{N}_{0}=\mathcal{N} \backslash \mathcal{N}_{1} \backslash\{j, k\} \\
& \text { if } \frac{\gamma_{j} / h_{j}-\gamma_{k} / h_{k}}{g_{k} / h_{k}-g_{j} / h_{j}}>\frac{\gamma_{j} / h_{j}-\gamma_{i} / h_{i}}{g_{i} / h_{i}-g_{j} / h_{j}}, \exists i \in \mathcal{N}_{1} \text { or } \frac{\gamma_{j} / h_{j}-\gamma_{k} / h_{k}}{g_{k} / h_{k}-g_{j} / h_{j}}<\frac{\gamma_{j} / h_{j}-\gamma_{i} / h_{i}}{g_{i} / h_{i}-g_{j} / h_{j}}, \exists i \in \mathcal{N}_{0} \text { then } \\
& \quad j=0, k=0
\end{aligned}
$$

\section{end if}

Output: $j, k, \mathcal{N}_{1}, \mathcal{N}_{0}$ 
Fig. 2 shows and compares the maximum sum ergodic capacity under PTP+PIP, PTP+AIP and $\mathrm{PTP}+\mathrm{ATP}+\mathrm{PIP}+\mathrm{AIP}$ constraints versus $P^{p k}$ where $P^{p k}=P_{i}^{p k}, \forall i$ is assumed. It can be seen from the figure that the maximum sum ergodic capacity achieved under PTP+AIP is larger than that achieved under PTP+PIP for any given $P^{p k}$. This is due to the fact that the AIP constraint is more favorable than the PIP constraint from SUs' perspective, since the former allows for more flexibility for SUs to allocate transmit power over different channel fading states. It is also observed that the performance under PTP+ATP+PIP+AIP is very close to that under PTP+PIP that is because the PTP constraint dominates over the ATP, PIP, and AIP constraints for all values of $P^{p k}$.

Fig. 3 shows the maximum sum ergodic capacity under ATP+PIP, ATP+AIP and PTP+ATP+ PIP+AIP constraints versus $P^{a v}$ where $P^{a v}=P_{i}^{a v}, \forall i$ is assumed. The maximum achievable sum ergodic capacity achieved under ATP+AIP is larger than that achieved under ATP+PIP for all values of $P^{a v}$ since the PIP constraint is stricter than the AIP constraint. The sum ergodic capacity under PTP+ATP+PIP+AIP is much smaller than that under ATP+PIP and ATP+AIP due to the fact that the PTP constraint is dominant over other constraints for all values of $P^{a v}$.

Fig. 4 shows the maximum sum ergodic capacity under PTP+PIP, ATP+PIP and PTP+ATP+ $\mathrm{PIP}+\mathrm{AIP}$ constraints versus $Q^{p k}$. It can be seen from the figure that the maximum sum ergodic capacity achieved under ATP+PIP is larger than that achieved under PTP+PIP for any given $Q^{p k}$. This is because the power allocation is more flexible for SUs under the ATP constraint than under the PTP constraint. The sum ergodic capacity under PTP+ATP+PIP+AIP saturates earlier than that under PTP+PIP and ATP+PIP, because it is restricted by the AIP constraint.

Fig. 5 shows the maximum sum ergodic capacity under PTP+AIP, ATP+AIP and PTP+ATP+ PIP+AIP constraints versus $Q^{a v}$. Due to the same reasons as for the results in Fig. 4, the sum ergodic capacity achieved under ATP+AIP is larger than that achieved under PTP+AIP. The sum ergodic capacity under PTP+ATP+PIP+AIP saturates earlier than that for PTP+AIP and ATP+AIP because of the presence of the PIP constraint.

Finally, Fig. [6 shows the maximum sum ergodic capacity under PTP+PIP, PTP+AIP, ATP+PIP, $\mathrm{ATP}+\mathrm{AIP}$ and PTP+ATP+PIP+AIP versus $W$. Similar performance comparison results as in the previous figures can be observed. One difference is that the sum ergodic capacities do not saturate with the increase of $W$. 


\section{CONCLUSION}

A cognitive radio network where multiple SUs share the licensed spectrum of a PU using the FDMA scheme has been considered. The maximum achievable sum ergodic capacity of all the SUs has been studied subject to the total bandwidth constraint of the licensed spectrum and all possible combinations of the peak/average transmit power constraints at the SUs and interference power constraint imposed by the PU. Optimal bandwidth allocation has been derived in each channel fading state for any given power allocation. Using the structures of the optimal power allocations under each combination of the power constraints, algorithms for finding the optimal power allocations have been developed too.

\section{REFERENCES}

[1] J. Mitola, "Cognitive radio: An integrated agent architecture for software defined radio,", Ph.D. dissertation, KTH, Stockholm, Sweden, Dec. 2000.

[2] S. Haykin, "Cognitive radio: Brain-empowered wireless communications," IEEE J. Sel. Areas Commun., vol. 23, pp. 201220, Feb. 2005.

[3] Q. Zhao, L. Tong and S. Ananthram, "Decentralized cognitive MAC for opportunistic spectrum access in ad hoc networks: A POMDP framework," IEEE J. Sel. Areas Commun., vol. 25, pp. 589-600, Apr. 2007.

[4] Y. Chen, Q. Zhao, and S. Ananthram, "Joint design and separation principle for opportunistic spectrum access in the presence of sensing errors," IEEE Trans. Inform. Theory, vol. 54, pp. 2053-2071, May. 2008.

[5] S. Ahmad, M. Liu, T. Javidi, Q. Zhao, and B. Krishnamachari, "Optimality of myopic sensing in multichannel opportunistic access," IEEE Trans. Inform. Theory, vol. 55, pp. 4040-4050, Sep. 2009.

[6] N. B. Chang and M. Liu, "Optimal channel probing and transmission scheduling for opportunistic spectrum access," IEEE Trans. Networking, vol. 55, pp. 4040-4050, Sep. 2009.

[7] Q. Zhao and B. M. Sadler, "A survey of dynamic spectrum access," IEEE Signal Process. Mag., vol. 24, pp. 79-89, May 2007.

[8] K. T. Phan, S. A. Vorobyov, N. D. Sidiropoulos, and C. Tellambura, "Spectrum sharing in wireless networks via QoS-aware secondary multicast beamforming," IEEE Trans. Signal Process., vol. 57, pp. 2323-2335, June 2009.

[9] R. Zhang and Y.-C. Liang, "Exploating multi-antennas for oportunistic spectrum sharing in cognitive radio networks," IEEE J. Sel. Topics Signal Process., vol. 2, no. 1, pp. 88-101, Feb. 2008.

[10] R. Zhang, "On peak versus average interference power constraints for protecting primary users in cognitive radio networks," IEEE Trans. Wireless Commun., vol. 8, pp. 2112-2120, Apr. 2009.

[11] R. Zhang and Y.-C. Liang, "Investigation on multiuser diversity in spectrum sharing based cognitive radio networks," IEEE Commun. Letters, vol. 14, pp. 133-135, Feb. 2010.

[12] G. Zheng, K.-K. Wong, and B. Ottersten, "Robust cognitive beamforming with bounded channel uncertainties," IEEE Trans. Signal Process., vol. 57, pp. 4871-4881, Dec. 2009.

[13] A. Ghasemi and E. S. Sousa, "Fundamental limits of spectrum-sharing in fading environments," IEEE Trans. Wireless Commun., vol. 6, pp. 649-658, Feb. 2007. 
[14] L. Musavian and S. Aissa, "Capacity and power allocation for spectrum-sharing communications in fading channels", IEEE Trans. Wireless Commun., vol. 8, pp. 148-156, Jan. 2009.

[15] X. Kang, Y.-C. Liang, A. Nallanathan, H. K. Garg, and R. Zhang, "Optimal power allocation for fading channels in cognitive radio networks: Ergodic capacity and outage capacity," IEEE Trans. Wireless Commun., vol. 8, pp. 940-950, Feb. 2009.

[16] R. Zhang, S. Cui, and Y.-C. Liang, "On ergodic sum capacity of fading cognitive multiple-access and broadcast channels," IEEE Trans. Inform. Theory, vol. 55, pp. 5161-5178, Nov. 2009.

[17] W. Yu and J. M. Cioffi, "FDMA capacity of a Gaussian multiple access channel with ISI," IEEE Trans. Commun., vol. 50, pp. 102-111, Jan. 2002.

[18] K. Kumaran and H. Viswanathan, "Joint power and bandwidth allocation in downlink transmission," IEEE Trans. Wireless Commun., vol. 4, pp. 1008-1016, May 2005.

[19] J. Acharya and R. D. Yates, "Dynamic spectrum allocation for uplink users with heterogeneous utilities," IEEE Trans. Wireless Commun., vol. 8, pp. 1405-1413, Mar. 2009.

[20] J. Gao, S. A. Vorobyov, and H. Jiang, "Cooperative resource allocation games under spectral mask and total power constraints," IEEE Trans. Signal Processing, vol. 58, to be published in Aug. 2010. (see also arXiv: CS.IT/0911.2847)

[21] I. Maric and R. D. Yates, "Bandwidth and power allocation for cooperative strategies in gaussian relay networks," IEEE Trans. Inform. Theory, vol. 56, pp. 1880-1889, Apr. 2010.

[22] X. Gong, S. A. Vorobyov, and C. Tellambura, "Joint bandwidth and power allocation in multi-user decode-and-forward relay networks," in Proc. 35th IEEE Inter. Conf. Acoustics, Speech, and Signal Processing, Dallas, TX, USA, Mar. 2010, pp. 2498-2501.

[23] X. Gong, S. A. Vorobyov, and C. Tellambura, "Joint bandwidth and power allocation with admission control in wireless multi-user networks with and without relaying," submitted to IEEE Trans. Signal Process..

[24] S. Boyd and L. Vandenberghe. Convex Optimization. Cambridge University Press, 2004. 


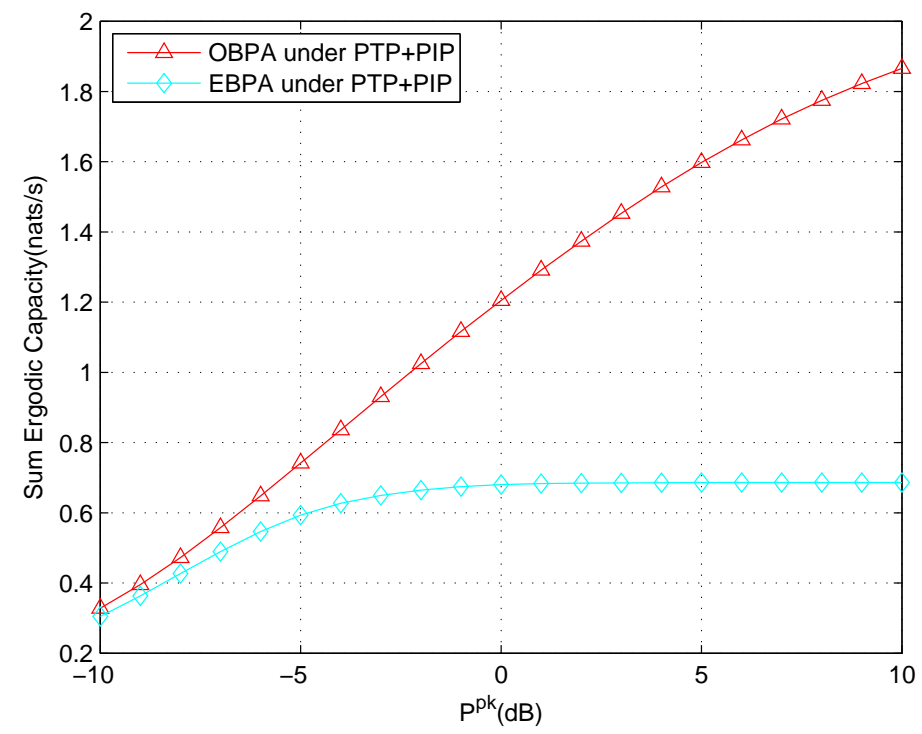

Fig. 1. Sum ergodic capacity vs $P^{p k}$.

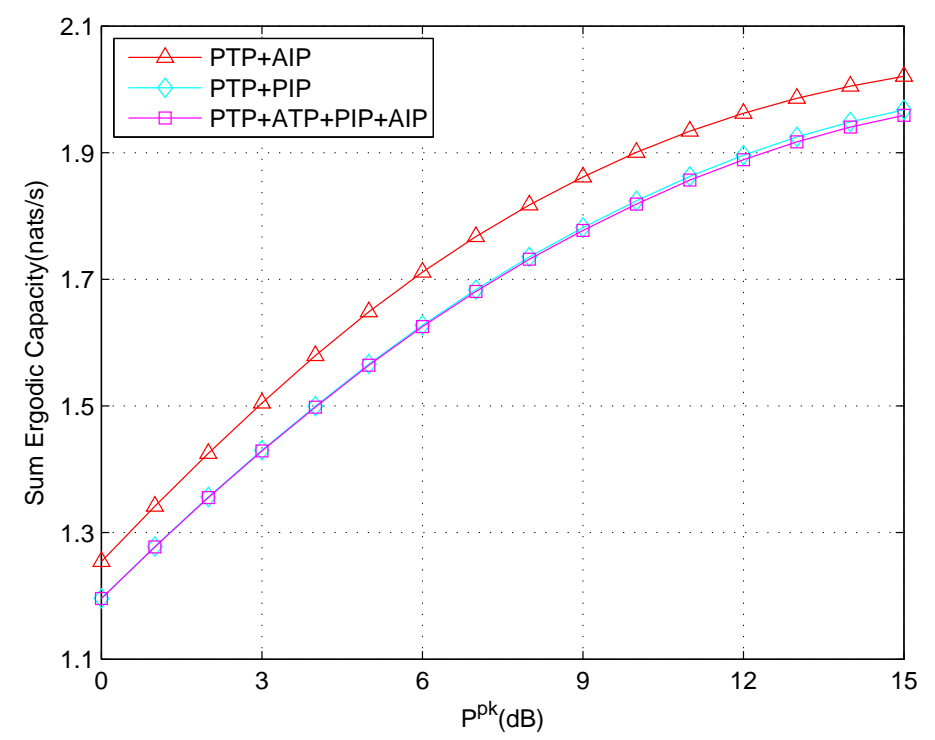

Fig. 2. Sum ergodic capacity vs $P^{p k}$. 


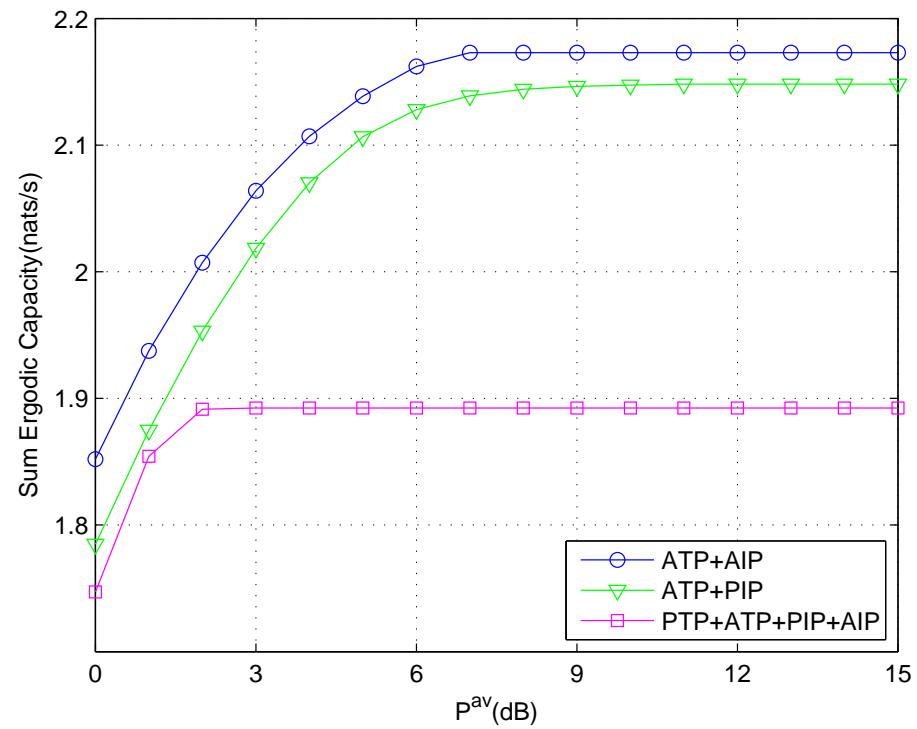

Fig. 3. Sum ergodic capacity vs $P^{a v}$.

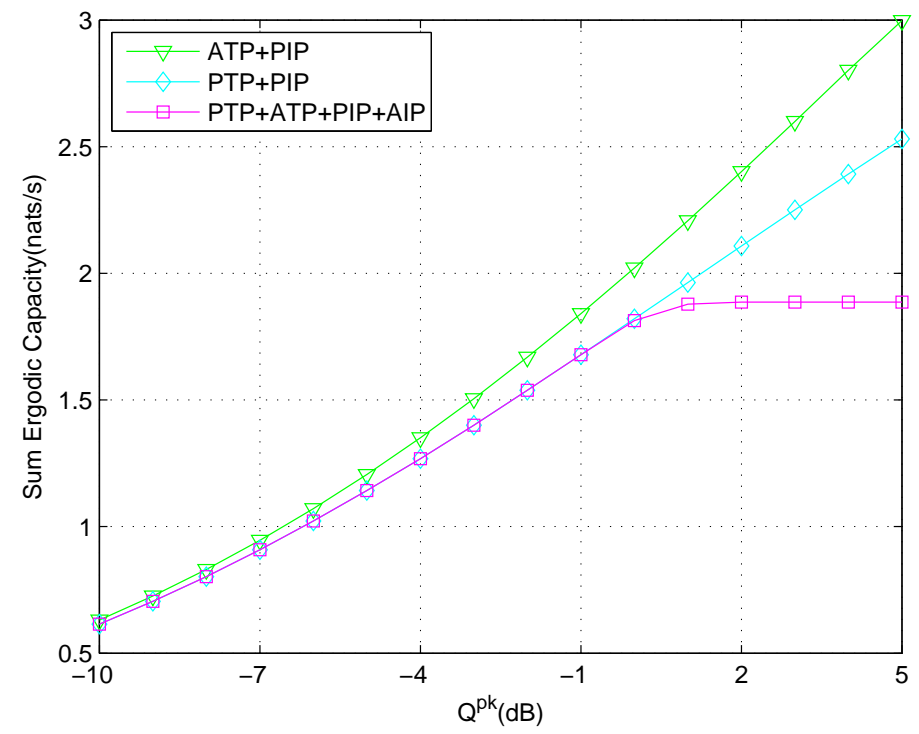

Fig. 4. Sum ergodic capacity vs $Q^{p k}$. 


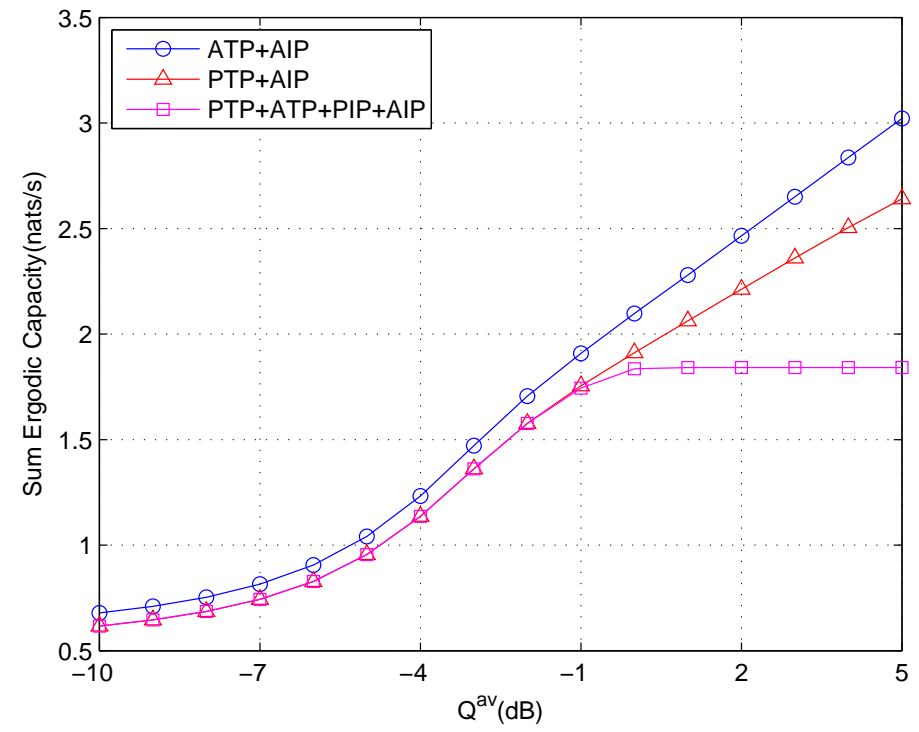

Fig. 5. Sum ergodic capacity vs $Q^{a v}$.

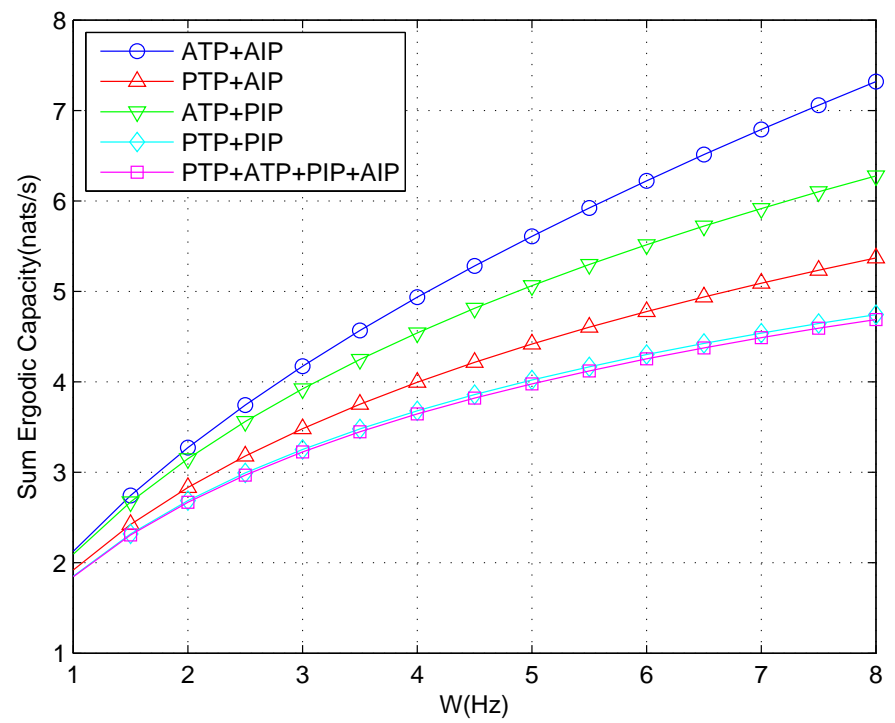

Fig. 6. Sum ergodic capacity vs $W$. 